\title{
Controlling the release of indomethacin from glass solutions layered with a rate controlling membrane using fluid-bed processing. Part 1: Surface and cross-sectional chemical analysis
}

Aswin Dereymaker ${ }^{1}$, David J. Scurr² ${ }^{2}$, Clive J. Roberts², Guy Van den Mooter $^{1}$

${ }^{1}$ Drug Delivery and Disposition, KU Leuven, Campus Gasthuisberg O\&N2, Herestraat 49, Box 921, 3000 Leuven, Belgium

${ }^{2}$ Laboratory of Biophysics and Surface Analysis, School of Pharmacy, The University of Nottingham, University Park, Boots Science Building, Nottingham NG7 2RD, United Kingdom

Corresponding author: Guy Van den Mooter - guy.vandenmooter@pharm.kuleuven.be

Address: Drug Delivery \& Disposition, Campus Gasthuisberg O\&N 2, Box 921, Herestraat 49, 3000 Leuven, Belgium. Tel.: +32 16 330304; fax: +32 16330305 


\section{List of abbreviations:}

API: active pharmaceutical ingredient

BCS: biopharmaceutics classification system

mDSC: modulated differential scanning calorimetry

EC: ethyl cellulose

ERL: Eudragit RL ${ }^{\circledR}$

INDO: indomethacin

PVP: polyvinylpyrrolidone

SEM: scanning electron microscopy

TEC: triethyl citrate

Tg: glass transition temperature

ToF-SIMS: time-of-flight secondary ion mass spectrometry

XRPD: X-ray powder diffraction 


\begin{abstract}
Fluid bed coating has been shown to be an adequate manufacturing technique to formulate poorly soluble drugs in glass solutions. Layering inert carriers with a drug-polymer mixture enables these beads to be immediately filled into capsules, thus avoiding additional, potentially destabilizing, downstream processing. In this study fluid bed coating is proposed for the production of controlled release dosage forms of glass solutions by applying a second, rate controlling membrane on top of the glass solution. Adding a second coating layer adds to the physical and chemical complexity of the drug delivery system so a thorough understanding of the physical structure and phase behavior of the different coating layers is needed. This study aimed to investigate the surface and cross-sectional characteristics (employing SEM and ToFSIMS) of an indomethacin-polyvinylpyrrolidone (PVP) glass solution, top-coated with a release rate controlling membrane consisting of either ethyl cellulose or Eudragit RL. The implications on the addition of a pore former (PVP) and the coating medium (ethanol or water) were also considered. In addition, polymer miscibility and the phase analysis of the underlying glass solution were investigated.
\end{abstract}

Significant differences in surface and cross sectional topography of the different rate controlling membranes or the way they are applied (solution vs. dispersion) were observed. These observations can be linked to the polymer miscibility differences. The presence of PVP was observed in all rate controlling membranes, even if it is not part of the coating solution. This could be attributed to residual powder presence in the coating chamber. The distribution of PVP among the sample surfaces depends on the concentration and the rate controlling polymer used. Differences can again be linked to polymer miscibility. Finally, it was shown that the underlying glass solution layer remains amorphous after coating of the rate controlling membrane, whether formed from an ethanol solution or an aqueous dispersion. 


\section{Introduction}

Although the potential of solid dispersions to increase the apparent solubility/dissolution rate and consequently the bio-availability of biopharmaceutics classification system (BCS) class II drugs has been widely demonstrated in the last couple of decades, there is still a huge discrepancy in the research input and the commercially available output. The main challenges of commercializing solid dispersions remain the long term physical stability of such systems, with the amorphous or molecularly dispersed drug inherently prone to phase separation, crystallization and ultimately the decrease in solubility. Not only can long term stability issues potentially arise, processing solid dispersion powders into their final dosage form can also lead to phase separation, as recently shown by Worku et al. during the compression of Naproxen polyvinylpyrrolidone (PVP) solid dispersions ${ }^{1}$. This is a hurdle which can be overcome by coating solid dispersions onto inert carriers and thus surpassing major additional downstream processing steps.

Coating glass solutions onto inert carriers can also be exploited to transform them into controlled release formulations. Coated pellets in the size range of $100 \mu \mathrm{m}-1 \mathrm{~mm}$ for controlled release purposes have already been demonstrated as beneficial as compared to controlled release coated tablets. This is thought to be as they are less prone to variability in stomach emptying rates in the fasted $s^{2} t^{2}$. In the fed state there are additional factors to take into consideration including the composition and caloric value of the administered meal and the size and density of the particles. Therefore, no real consensus has been reached yet on stomach residence times of controlled release formulations. Pellets also show a more even spread in the gastrointestinal tract in comparison with a single coated tablet. Having a high number of coated pellets also reduces the risk for dose-dumping ${ }^{3,4}$. Furthermore, the surface of amorphous solid dispersion formulations has also been shown to be more vulnerable to crystallization of the amorphous drug phase ${ }^{5}$, hence an additional coating layer could potentially stabilize these formulations.

The first and foremost reason to produce a controlled release formulation from glass solutions is to maximize the absorption window for poorly soluble drugs by allowing an appropriate amount of dissolved drug to be available for absorption at extended time intervals. Moreover, a slower release rate will reduce the precipitate rate of poorly soluble drugs from their supersaturated state. Controlling the release of glass solutions also allows for a decreased dosing scheme, a better patient compliance and a reduced risk of side effects ${ }^{6}$. It could, however, be 
argued that poorly soluble drugs already inherently possess a slow release dissolution profile, but this is compound specific, and non-adjustable.

The overarching goal of this study is to investigate the potential of coated glass solutions to control or reduce the release rate of poorly soluble drugs. For this purpose, bilayer coated sucrose carriers (pellets), of which the first layer consists of a glass solution of indomethacin (INDO) in polyvinylpyrrolidone K25 (PVP) in a 30:70 (w:w) ratio were generated. To tune the release of indomethacin from this glass solution, a top layer was applied, consisting of the insoluble polymer ethyl cellulose (EC) and the insoluble but swellable polymer Eudragit RL ${ }^{\circledR}$ (ethyl acrylate: methyl methacrylate: trimethylammonioethylmethacrylate co-polymer in a molar ratio of 1:2:0.2) (ERL).

Owing to the complex composition of the coated glass solutions, typical solid state analytical techniques such as modulated differential scanning calorimetry $(\operatorname{mDSC})^{7}$ or X-ray powder diffraction (XRPD) are inadequate for this specific multi-layer samples because they only provide bulk analysis (no spatial resolution). Thermal analysis by (m)DSC can detect two separate amorphous phases, provided they are larger than the critical length scale of the technique $(\sim 30 \mathrm{~nm})^{8}$. In conventional wide angle XRPD, transmission geometry doesn't allow depth resolution as incident $\mathrm{x}$-rays go completely through the sample. Also, in reflection geometry, the penetration depth of the x-rays doesn't allow in depth resolution, spatial resolution can only be achieved in combination with other techniques ${ }^{9}$. Glancing angle $\mathrm{x}$-ray techniques can be used to measure varying sample thicknesses since penetration depths are lower and can be calculated according to the incident $\mathrm{x}$-ray beam angle ${ }^{10,11}$. This leads to conclude that mDSC and/or XRPD don't provide adequate information on the different layers of complex coated systems. It has even been shown recently that the phase behavior study of a single layer glass solution onto an inert carrier is not straightforward ${ }^{12}$.

ToF-SIMS has been used as a surface analysis technique in a wide array of research fields; from biological samples (cells, tissues, proteins, lipids...) ${ }^{13-17}$, over material science $^{18}$ to pharmaceutical formulations ${ }^{19-24}$. ToF-SIMS is based upon the bombardment of a sample surface with a primary ion beam (e.g. $\mathrm{Ar}^{+}, \mathrm{Cs}^{+}, \mathrm{Bi}_{3}{ }^{+}$) under ultra-high vacuum conditions. The impact of the kinetic energy of these primary ions upon the sample surface will result in desorption of electrons, atoms, molecular fragments and whole molecules. The ionized molecular fragments are of particular interest for ToF-SIMS. These secondary ions are accelerated and injected into the Time-of-Flight analyzer. For ions with an equal charge, this 
will result in equal kinetic energy and thus the velocity of these ions will depend on their massto-charge ratio $(\mathrm{m} / \mathrm{z})$. Hence, the time needed to reach the detector is indicative for the $\mathrm{m} / \mathrm{z}$ of the detected molecular fragment and will result in a negative or positive mass spectrum, depending on the charge of the collected molecular fragments ${ }^{19,20,25-27}$ ToF-SIMS has a very low detection limit (ppb range), a high surface sensitivity and high spatial resolution $(0.2 \mu \mathrm{m})$ ${ }^{28}$. Owing to these properties, ToF-SIMS is highly suited for surface chemical identification and surface chemical distribution (mapping) and will therefore be used to analyze the layered beads, and by doing so, elucidate their chemical structure.

The purpose of this study ("part 1") is to investigate the phase behavior of the surface and physical cross-sections of sucrose beads coated with two layers: an inner layer made up of a glass solution of indomethacin in PVP K25 and an outer layer consisting of a rate controlling membrane made up of ethylcellulose or Eudragit RL. In addition, the influence of a pore former (PVP K25) in the outer layer and application of the outer layer from an organic solution or an aqueous dispersion on the phase behavior was investigated. Surface and cross-sectional morphology was investigated by scanning electron microscopy (SEM), the chemical composition and distribution of the bead surfaces and cross-sections was analyzed using ToFSIMS. Physical structure of the glass solutions was assessed by XRPD, while polymer mixing was tested using mDSC. 


\section{Materials and Methods}

\section{Materials}

Indomethacin was purchased from FAGRON Ltd. (Waregem, Belgium). Polyvinylpyrrolidone K 25 was a generous gift from BASF (Ludwigshafen, Germany). Sucrose spheres (diameter $710-850 \mu \mathrm{m}$ ) were kindly donated by Hanns G. Werner GmbH (Tornesch, Germany). Ethyl cellulose (ethoxy content $48.0-49.5 \% \mathrm{w} / \mathrm{w}$ ) powder and triethyl citrate were purchased from Sigma-Aldrich (Zwijndrecht, The Netherlands). Eudragit RL ${ }^{\circledR} \mathrm{PO}$ and $30 \mathrm{D}$ were purchased from Evonik Industries (Darmstadt, Germany).

\section{Methods}

\section{Fluid bed coating}

Coated beads were prepared using an Aeromatic MP 1 multiprocessor (GEA, Bubendorf, Switzerland) in a bottom spray setup, equipped with a Würster insert. A 30 : 70 (w:w ) INDOPVP (w/w) glass solution with a total solid content of 250,0 g was coated onto 500,0 $\mathrm{g}$ of sucrose beads from a $10 \%$ (w/v) ethanol solution. The sucrose spheres were loaded into the preheated coating chamber at $50{ }^{\circ} \mathrm{C}$ and heated for 10 minutes. The drug-polymer solution was coated onto the sucrose pellets at a feed rate of $13 \mathrm{~cm}^{3} / \mathrm{min}$ and this feed was atomized at an air pressure of 1.5 bar. Meanwhile the heated air stream was passing through the fluid bed coater at a rate of $1.78 \mathrm{~m}^{3} / \mathrm{min}$. When the spraying was finished, the pellets were dried until immobilization due to electrostatic charge was observed. The coated spheres where unloaded, weighed and dried for an additional 48 hours in an oven at $50{ }^{\circ} \mathrm{C}$. In the case when a top layer (rate controlling membrane) was applied, the feed solution was immediately changed after completion of the glass solution layer. The controlled release top layer consisted of a rate controlling polymer (ERL or EC) which was applied from a 10\% w/v ethanolic solution. Additional batches were prepared that contained PVP K25 as a pore former in the rate controlling membrane (in a concentration of $10 \%$ or $25 \% \mathrm{w} / \mathrm{w}$ relative to the total solid content) or the plasticizer TEC, added in a concentration of $20 \% \mathrm{w} / \mathrm{w}$ relative to the amount of rate controlling polymer. Finally ERL was also applied as an aqueous dispersion (10\% w/v) instead of an ethanolic solution. Controlled release top coating total solid content was $200,0 \mathrm{~g}$. The coating process parameters are the same for the top coating layer as for the glass solution layer, except for the feed rate with the ERL ethanolic solutions. Here the feed rate was reduced to $6.5 \mathrm{ml} / \mathrm{min}$ because of the electrostatic charges created inside of the fluid bed coater. After 
completion of the coating, the beads were also dried in the coater for at least 10 minutes, removed from the coater and additionally dried in an oven for at least 48 hours. Ethanolic solutions are dried at $50^{\circ} \mathrm{C}$, aqueous dispersions at $60^{\circ} \mathrm{C}$ to allow for curing of ERL.

\section{Spray drying}

The miscibility between PVP K25 and EC or ERL was investigated for spray dried samples. ERL or EC were combined with PVP K25 in different ratios: 100-0, 75-25, 50-50, 25-75 and 0-100 \% w/w. Each composition was spray dried from a 10\% w/v ethanol solution using a Büchi Mini Spray Dryer B-191 (Büchi, Flawil, Switzerland). The inlet air temperature was $50^{\circ} \mathrm{C}$, the heated air flow rate $0,56 \mathrm{~m}^{3} / \mathrm{min}$, the atomizing air flow rate $0.02 \mathrm{~m}^{3} / \mathrm{min}$ and the feed rate $4.8 \mathrm{~cm}^{3} / \mathrm{min}$. The obtained polymer mixtures were additionally dried for 48 hours in a vacuum oven at $25^{\circ} \mathrm{C}$ (Mazzali Systems, Monza, Italy), prior to $\mathrm{mDSC}$ analysis to investigate polymer miscibility.

\section{Scanning electron microscopy}

The morphology of the coated beads was investigated with SEM using a Philips XL30 ESEMFEG instrument (Philips, Eindhoven, The Netherlands) equipped with a field emission electron gun. Whole beads and cross sections (made with a scalpel under an optical microscope) were fixed on an aluminum stub using double-sided carbon tape. The samples were coated with gold by sputtering for $45 \mathrm{~s}$ at $20 \mathrm{~mA}$. The SEM was used with an acceleration voltage of $2.00 \mathrm{kV}$, a spot size of 3 and a secondary electron detector.

\section{Time-of-flight secondary ion mass spectrometry}

For chemical composition and distribution analysis, time-of-flight secondary ion mass spectrometry (ToF-SIMS) was performed using a ToF-SIMS IV (ION-TOF Gmbh, Münster, Germany). This spectrometer was equipped with a pulsing bismuth liquid metal gun $\left(\mathrm{Bi}_{3}{ }^{+}\right)$and a single stage reflectron analyzer. A flood gun was applied to produce low energy electrons to compensate surface charging from the primary ion beam (positive charges). For surface analysis, samples were fixed to glass slides using double sided tape prior to analysis. When analyzing whole beads, only the uppermost part of the bead was analyzed due to the shape and size of the beads. This surface analysis comprised of an analysis area of $200 \times 200 \mu \mathrm{m}$ with a raster scanned resolution of $256 \times 256$ pixels. For cross-sectional analysis, beads were embedded into Epofix ${ }^{\mathrm{TM}}$ cold-setting resin, and sliced using a glass knife on a RMC ultramicrotome PowerTome. For the cross-sectioned beads, an analysis area of $500 \times 500 \mu \mathrm{m}$ 
was raster scanned with a resolution of $256 \times 256$ pixels. All samples were analyzed in the negative ion polarity mode and analyzed using SurfaceLab 6 (ION-TOF Gmbh, Münster, Germany). Firstly, controls were analyzed with reference material of all components. Different characteristic ions were chosen as the most selective to the specific compound. The chosen indomethacin marker was $\mathrm{C}_{7} \mathrm{H}_{10} \mathrm{O}_{4}$, , the PVP marker was $\mathrm{C}_{3} \mathrm{H}_{4} \mathrm{~N}_{2} \mathrm{O}^{-}$, the EC marker was $\mathrm{C}_{2} \mathrm{H}_{5} \mathrm{O}^{-}$, the ERL marker $\mathrm{CH}_{3} \mathrm{O}$ - and the sucrose marker $\mathrm{C}_{12} \mathrm{H}_{21} \mathrm{O}_{11^{-}}$. Marker intensities and ion peaks for the control samples are shown in Figure A of the supplementary data. Measured secondary ion intensities for every component were normalized to total intensity count to allow for a semi-quantitative comparison between samples. It should be noted that the control ion for EC is also formed in the ERL control spectrum. Since there are no samples where both polymers are present at the same time, this didn't pose any interpretation problems.

\section{Modulated differential scanning calorimetry}

mDSC analysis of spray dried polymers and polymer mixtures was carried out with a TA instruments Q2000 modulated DSC (Leatherhead, UK) equipped with a refrigerated cooling system (RCS90). The DSC cell was purged with a nitrogen flow of $50 \mathrm{ml} / \mathrm{min}$ during analysis. Data processing was performed using TA Instruments Universal Analysis software (version 4.4, Leatherhead, UK). TA Instruments standard aluminium pans (Brussels, Belgium) were used for all measurements. All sample masses were between 5-6 mg (accurately weighed). The samples were heated from $0^{\circ} \mathrm{C}$ to $180^{\circ} \mathrm{C}$. A heating rate of $2^{\circ} \mathrm{C} / \mathrm{min}$ was applied with a temperature modulation of $0.636^{\circ} \mathrm{C}$ every $40 \mathrm{~s}$. All samples were measured in triplicate. Glass transition temperatures were measured at half height in the reversing heat flow. The step jump in heat capacity observed in the reversing heat flow signal was further examined in the corresponding derivative signal after Savitsky-Golay smoothing with points of window set at $10^{\circ} \mathrm{C}$. n-Octadecane and indium were used to calibrate and validate the DSC temperature scale. Indium was also used to calibrate and validate the enthalpic response. The heat capacity was calibrated and validated using sapphire disks.

\section{X-ray powder diffraction}

Coated beads and reference powders were analyzed at room temperature using an automated X'pert PRO diffractometer (PANalytical, Almelo, the Netherlands). All samples were placed in the sample holders, clamped between Kapton foil and analyzed in transmission mode using a $\mathrm{Cu}$ tube ( $\mathrm{Ka} 1.5418$ Angstrom; generator at $45 \mathrm{kV}$ and $40 \mathrm{~mA})$. Analysis was performed in continuous scan mode in $2 \theta$ range from $4^{\circ}$ to $40^{\circ}$ with a $0.0167^{\circ}$ step size and 200 seconds 
counting time per step. The X'pert Data Collector and the X'pert Data Viewer (PANalytical, Almelo, the Netherlands) were used for data collection and analysis. 


\section{Results}

\section{Surface and cross sectional investigation of the coated beads with SEM}

The structures of the outer surface of whole beads with different outer coatings are shown in Figure 1. Figure 1A shows an INDO-PVP glass solution coated bead which appears spherical in shape with a smooth surface. This bead exhibits surface cracks with different lengths and depths. Right below the largest crack, there is one major dent in the coating. Figure 1B shows beads which were coated with an inner layer of INDO-PVP and an outer layer of EC. These beads are spherical but with no cracking observed. The surface appears slightly rougher compared to the INDO-PVP surface. An INDO-PVP glass solution inner layer combined with an ERL outer layer, coated from an ethanol solution, is displayed in Figure 1C. No cracks were observed but now the surface is rough and shows pitted 'golf ball-like' surface structure. Finally, Figure 1D presents an INDO-PVP glass solution layer with an ERL top coating sprayed from an aqueous dispersion. This resulted in smooth, spherical beads with a few rougher spots. A few very minor cracks or crescent shaped holes can be observed.

The SEM analysis of the cross-sectioned beads revealed the different coating layers and inner morphology of the different formulations as illustrated in Figure 2. Figure 2A depicts a cross section of the INDO-PVP coated bead. There is a distinct difference in morphology between the inner sugar core, which has a rough morphology, and the glass solution coating which has a smoother appearance. There are also cracks visible in the cross-sectioned view. Figure $2 \mathrm{~B}$ and 2B' show the sugar core, INDO-PVP inner layer and EC outer layer. The sugar core and glass solution layer possess the same features as described in the previous figure. The EC outer coating layer has a distinct 'layered' structure and seems to be loosely attached to the inner glass solution layer with a distinct gap between the two coating layers. Beads with a top coating of EC and $20 \% \mathrm{w} / \mathrm{w}$ of TEC were also analyzed (Figure B of the supplementary data), but no differences were observed when compared to Figure $2 \mathrm{~B}$ and $2 \mathrm{~B}^{\prime}$. The EC layers are morphologically very different from the ERL top coating, which is shown in Figures $2 \mathrm{C}$ and $2 C^{\prime}$, where it is sprayed from an ethanol solution and in Figure 2D from an aqueous dispersion. The ERL layer is much smoother compared to the EC layer and equally smooth compared to the glass solution layer but with the absence of cracks. In this case, the two different coating layers are also attached to each other.

\section{Chemical surface composition and distribution of the coated beads by ToF-SIMS}


Using the data obtained from the control samples, sample spectra were investigated for the presence or absence of each marker upon the samples. Secondary ion images are then constructed for chosen ions. ToF-SIMS secondary ion spectra and images of the surface of the INDO-PVP glass solution layer are shown in Figure 3. Figure 3a Shows the presence of ion peaks at the marker positions of PVP (left) and INDO (right), Figure $3 \mathrm{~b}$ shows, from left to right, the total ion image, the PVP marker ion image, the INDO ion marker image and the overlay of the PVP (green) and INDO (blue) marker ion. From the ion spectra (Figure 3a), it is apparent that both molecules are present in the glass solution coating. From the total ion image, the cracks in the surface are also visible. In terms of distribution, by observing the markers of INDO, PVP and the overlay of both, it is clear that the presence of both molecules along the entire surface shows a homogeneous distribution.

Secondary ion images of ERL coated formulations are provided in Figure 4. Figure 4a-d show ion images of ERL 100\%, ERL-PVP 90-10\%, ERL-PVP 75-25\% and ERL latex 100\% respectively. Secondary ion spectra are provided in Figure $\mathrm{C}$ of the supplementary data. All ERL top coated formulations show presence of PVP marker ion on the sample surface, as can be seen in in the secondary ion spectra. The presence of PVP is expected for the ERL-PVP 90$10 \%$ and ERL-PVP 25\% coatings, because PVP is used here as a pore former in the rate controlling membrane, but it was not anticipated in coatings made up of pure ERL. Although all of the formulations show PVP presence at the surface, the distribution is different. From the ion images in Figure 4 it can be seen that in the formulations where PVP is used as a pore former, the distribution of PVP is more homogeneous throughout the surface. In both ERL $100 \%$ samples, which contained no pore former, the secondary ion images show PVP 'hot spots', i.e. local areas with a high marker intensity of PVP (column 3 of Figure 4a and 4d) and a low marker intensity of ERL (column 2 of Figure 4a and 4d). Secondary ion spectra of ERL based samples also show a very small INDO marker intensity for ERL 100\% and ERL 100\% latex. For ERL-PVP 90-10\% and ERL-PVP 75-25\%, no clearly defined peaks could be observed in the spectra. This is also shown in the INDO marker ion images of Figure 4 where only very few localized INDO spots can be observed in ERL 100\% and ERL 100\% latex. ERLPVP 90-10\% and ERL-PVP 75-25\% only show background noise. It has to be remarked that not the entire surface is represented in the ion images of the sample as it was not possible to obtain data from the full $200 \times 200 \mu \mathrm{m}$ range. The system seems to cope with the topography but not with the curvature of the sample as it progressively goes out of focus. The loss of SIMS data due to the sample curvature is illustrated for each sample in the effect upon the total ion 
image which is provided for each system in column 1 of Figure 4. As the ToF-SIMS is a semiquantitative analysis method, only trends can be derived from the ion intensities obtained. To be able to compare different measurements and different formulations, the ion intensities presented were normalized by total ion intensity. Figure 5 illustrates the intensities for the PVP and ERL diagnostic ions. Although the ERL ion intensity decreases with decreasing ERL content in the outer layer, the same cannot be said of the PVP marker intensity. Surprisingly, there seems to be a similar PVP marker intensity in both 100\% ERL outer layers, either sprayed from an ethanol solution or aqueous dispersion, and ERL-PVP 90-10\%. Additionally, when PVP is added to the formulation as a pore former, a large variation between three independent measurements was observed. ERL-PVP 75-25\% shows the highest PVP marker intensity. Since no INDO marker peaks can be observed in ion spectra of ERL-PVP 90-10\% and ERL-PVP 75$25 \%$, the marker intensities for INDO in Figure 5 represent background noise, INDO marker intensities for ERL 100\% and ERL 100\% latex are barely larger than this background noise intensities.

Secondary ion spectra from the EC coated samples were also analyzed for marker ion intensities. The ion spectra of EC coated samples are shown in Figure D of the supplementary data. Secondary ion images were constructed from these spectra. These images are shown in Figure 6. In this figure, rows A, B, C and D represent the EC 100\% coated sample, EC-PVP 90-10\%, EC-PVP $75-25 \%$ and EC $100 \%$ with the addition of $20 \%$ TEC as a plasticizer (EC $100 \%$ - TEC) respectively. Once again, from the ion spectra, the presence of PVP can be observed in every sample, even in those samples that would not be anticipated to contain PVP in their outer layer, namely EC 100\% and EC 100\% - TEC. Local PVP regions are again visible, but this time they are even more pronounced and spread out diffusely along a large area of the analyzed surface. From the overlay ion images of the samples with PVP as a pore former (Figure $6 \mathrm{~b}$ and $6 \mathrm{c}$, column 4), it can be observed that the distribution of PVP is more heterogeneous, compared to both EC 100\% samples and also compared to ERL samples with identical pore former amounts. Ion peaks for the INDO marker ion can be distinguished in all EC samples (Figure D of the supplementary data), although they have very small intensities $\left(10^{-5}\right.$ range). Only in the EC-PVP 90-10\% sample, one specific INDO spot can be observed (Figure 6b, column 3). For the marker ion intensities of EC samples in Figure 7, the EC marker intensity is similar for EC 100\%, EC 100\% - TEC, and EC-PVP 90-10\%. EC-PVP 75-25\% has a decreased EC marker intensity. Contrary to ERL top layer samples, samples containing PVP as a pore former also have higher ion intensities for the PVP marker as compared to the $100 \%$ EC coated 
formulations. However, the ion intensity for the PVP marker is higher for the EC-PVP 90-10\% sample, compared to the EC-PVP 75-25\% sample. Similar to ERL samples, INDO marker intensity is very low and is for the most part background noise.

\section{Chemical cross-sectional composition and distribution of the coated beads by ToF-SIMS}

Cross sections of each bead formulation were also analyzed with ToF-SIMS. The same markers for all components were used plus an additional sucrose bead marker ion. Presence of all marker ion peaks was checked and secondary ion images are then constructed for these marker ions. ToF-SIMS secondary ion spectra are shown in Figure E of the supplementary data and images of the INDO-PVP glass solution cross-section are shown in Figure 8. From the distribution of the different marker ions, a well-defined INDO-PVP glass solution layer can be observed as an outer layer on top of the sucrose bead. It is interesting to note here that inside the sucrose bead core, there seems to be presence of INDO and PVP marker. This was also observed when a control sample was analyzed, as seen in the sucrose control sample ion intensities (supplementary data Figure F). This shows that the presence of INDO and PVP marker ions is inherent to the sucrose bead, which molecular constitution is not fully known, as they were purchased as such. Furthermore, the glass solution layer does not show a homogeneous distribution of INDO and PVP across the diameter of the bead. INDO seems to be more present at the inner part of the glass solution layer, while PVP is more present at the outer part of the glass solution layer. The presence of two sugar spots in the glass solution layer is probably due to chipping during cross-sectioning.

Secondary ion images of the cross-sections of ERL based outer layer formulations are provided in Figure 9. Figure 9a-d show ion images of cross-sections of sugar cores, coated with an INDOPVP glass solution and outer coating of ERL 100\%, ERL-PVP 90-10\%, ERL-PVP 75-25\% and ERL latex $100 \%$ respectively. Secondary ion spectra are provided in Figure $G$ of the supplementary data and show, not surprisingly ion peaks for all ion markers in every formulation. From the ion images it can be seen that INDO and PVP show a well-defined glass solution layer on top of the sucrose core. This glass solution layer in turn is again separated from the ERL top layers. PVP distribution along the ERL 100\% layer is not seen and is very diffuse along the ERL 100\% latex layer. As PVP is incorporated as a pore former, its intensity along the outer layer increased (Figure 9 column 3). In all formulations, INDO marker intensity is noticed solely in the glass solution layer, except for the ERL $100 \%$ latex layer where a INDO 
marker presence is observed right above the glass solution layer (Fig. 9d, Column 4). ERL marker presence is limited to the outer coating layer.

Cross-sections of EC based outer layer formulations were analyzed similarly to the ERL based outer layer formulations. Figure 10a-d provides ion images of the cross-sections of all different formulations, while all ion spectra are provided in Figure $\mathrm{H}$ of the supplementary data. Again, presence of all ion markers of the different components of the formulation was observed. Ion images show clearly defined INDO-PVP glass solution and EC based coating layers. PVP marker ion presence is observed in all EC coating layers, but is becoming more intense when applied as a pore former. This minor presence is attributed to the EC itself as can be seen from the control intensities (supplementary data Figure I), and is similar to the sucrose control mentioned earlier. A similar phenomenon is not observed for the INDO marker and has no visible marker intensity in the EC based top layer. The smearing of the outer EC $100 \%$ layer (Figure 10a, column 5) is a result of the bead cross-sectioning process.

In order to investigate if INDO or PVP can migrate into the outer ERL or EC based coating, this coating layer is divided into several regions of interest, forming an inner, middle and outer layer of the top coatings (One example of the EC 100\% sample is given in Figure J of the supplementary data). The different regions of interest intensities and total intensity of the INDO and PVP ion markers of the ERL top coatings are shown in Figure 11. Figure 11a represents PVP marker intensities and Figure $11 \mathrm{~b}$ shows INDO marker intensities. Identical intensity plots for EC top coatings are represented in Figure 12, where Figure 12a shows PVP marker intensities and Figure 12b represents INDO marker intensities. PVP marker intensities are higher in ERL (Fig. 11a) and EC (Fig.12a) coatings where PVP was used as a pore former, which confirms previous results from the ToF-SIMS surface analysis. All ERL top coating formulations show a PVP intensity gradient from the inner to the outer layer. INDO marker total intensity (Fig. 11a) is largest in ERL 100\% latex top coating. ERL 100\%, ERL-PVP 9010\% and ERL-PVP 75-25\% show similar INDO marker intensities. Similar to PVP marker intensities, there is an intensity gradient from the inner to the outer layer of all ERL based top coatings. A PVP marker intensity gradient is also observed in all EC formulations, except for EC-PVP 75-25\%. This gradient is less pronounced than the one in ERL formulations. An INDO marker gradient is also observed for all EC top coatings (Fig. 12b), except EC-PVP 75-25\%, but INDO marker intensities are slightly lower compared to ERL top coatings. The intensity difference is also most pronounced between the inner and middle layer of the EC based top coatings. The INDO marker intensity of the EC or ERL top coatings are still considerably small 
when compared to the marker intensity of the glass solution layer. This ranges from $4.5 \%$ for EC-PVP $75-25 \%$ to $14 \%$ for ERL-PVP $75-25 \%$ and ERL $100 \%$ latex.

\section{Polymer miscibility}

Polymer blends were prepared by spray drying from an ethanol solution and analyzed by mDSC to investigate polymer miscibility. Miscibility was evaluated based on the position and number of glass transition events. The glass transition temperature was measured in the reversing heat flow (half height in the heat capacity step change) and first derivative of the reversing heat flow (peak value). mDSC thermograms with these signals are presented in Figure 13 with ERL based samples in figure 13a and EC based samples in figure 13b. The glass transition temperatures of all analyzed samples are given in Table 1 (EC-based samples) and Table 2 (ERL-based samples). The mean glass transition temperature of spray dried pure PVP, EC and ERL is $156.8^{\circ} \mathrm{C}, 125.1^{\circ} \mathrm{C}$ and $53.5^{\circ} \mathrm{C}$ respectively. Two Tg's can be observed in the spray dried polymer blends, indicating that PVP is not miscible (or only to a certain extent) with ERL or EC. The major difference between EC-PVP mixtures and ERL-PVP relates to the fact that in the EC-PVP systems both Tg's remain more or less at the same temperature, while in coatings consisting of ERL-PVP both Tg's decrease with increasing PVP content. No glass transition event of the PVP fraction could be observed in the ERL-PVP 75-25 blend.

\section{Physical structure of the glass solution layer}

Different reference materials and coated beads were investigated by XRPD to investigate the stability of the solid state properties of the glass solution when applying a rate controlling membrane. Figure 14 shows the diffractograms of the reference materials, an INDO-PVP glass solution coating and different ERL based membrane top layers. All coated samples show the Bragg peaks from the sucrose pellets, but none of them show the typical Bragg peaks of $\gamma$ indomethacin, which is the most stable crystalline form. Representative indomethacin Bragg peaks are indicated with arrows at $2 \theta$ angles of $12.80^{\circ}$ and $17.35^{\circ}$. EC based samples were also analyzed and neither showed indomethacin Bragg peaks (data not shown). 


\section{Discussion}

\section{Coated bead surface and cross-sectional topography}

The SEM image of the INDO-PVP coated bead (Figure 1A) shows a smooth surface with cracks of different sizes running all along the surface. These cracks are proposed to a result of the drying of the glass solution. The spraying solvent, in this case ethanol, acts as a plasticizer of the INDO and PVP mixture, and as this solvent evaporates, the drug polymer mixture becomes more brittle as a result of an increasing glass transition temperature. This, together with contractional forces as a result of solvent evaporation on the spherical bead surface, could result in a cracked INDO-PVP glass solution layer. A cross-sectional image (Figure 2A) of these glass solution beads demonstrate that these cracks can extend from the outer surface to the sucrose core of the bead. These cracks also remain present when the INDO-PVP glass solution is top coated with an EC or ERL membrane (Figure 2B, 2B', 2C and 2C'). No cracks are present in any of the EC or ERL membranes themselves due to the probable higher flexibility of these films, which solely exist of polymer (or polymer mixtures). The topography of these coatings varies from very rough (ERL solution layer), over mildly rough (EC layer), to generally smooth (ERL dispersion layer). It is clear that the same polymer, sprayed from a solution or an aqueous dispersion generates vastly topographically different surfaces. This is not unexpected as the film forming process from an aqueous dispersion is significantly different from that of a solution. The beads coated with the dispersion were also additionally cured to further optimize polymer inter-diffusion and coalescence, and this probably contributes to the generation of a smooth surface.

Although the cross-sectioning procedure applied induced specific features (artifacts) on the surface of the cross section, like an unremoved part of the EC layer at the bottom part of Figure $2 \mathrm{~B}$ or cutting traces observed in the glass solution layer of Figure 2B', the difference between the glass solution layer with an additional ERL top layer or an EC top layer is very clear. Firstly, the EC membrane shows a layered structure along the coated layer, while the ERL membrane shows a smooth topography along the cross-section more resembling the glass solution layer. A second main difference lies in the attachment of the top layers to the glass solution layer. While the border between the ERL coating and the glass solution can only be observed through a change in grey tone, the border between the EC layer and the glass solution layer is indicated by the presence of a discernible delamination. It is impossible to know if this opening already existed before the cross sectioning or if it was created during cross sectioning. However, the fact that all samples were cut in the same way, suggests that the EC layer is, at the least, much 
more loosely attached compared to the ERL layer. The miscibility data, shown in the mDSC experiments, provide further arguments for this observation.

\section{Composition of coated bead surfaces}

ToF-SIMS surface analysis showed a clear determination of the composition and polymer distribution along the surface of the coated beads. We recently reported that INDO-PVP coated beads $(30-70 \% \mathrm{w} / \mathrm{w})$ are forming a glass solution, i.e. one phase systems characterized by a single $\mathrm{Tg}$ where the drug is molecularly dispersed into the polymer matrix ${ }^{12}$. The homogeneous distribution observed along the surface of INDO and PVP ions in the present study suggests the same excellent miscibility of both components. ToF-SIMS was able to clearly discriminate chemically between 4 components in total (ERL, EC, PVP and INDO) in samples which can contain up to 3 of these components at the same time. This was demonstrated by the components marker ions which showed a high specificity. In contrast to the expected presence and distribution of INDO and PVP in the glass solutions, EC and ERL layers showed unexpected results. Where its presence would not be anticipated, PVP markers are clearly observed in pure ERL or EC top coatings (Fig. 4a,d and 6a,d Column 3 respectively). There are two possibilities to explain the presence of PVP in these layers, the first one being a contamination with residual PVP, that became attached to the coating processor wall or Würster insert from the prior glass solution coating step. This is possible because the two coating layers are applied consecutively during the coating process, so there is always a fraction of droplets which are not sprayed on the beads and stick to the inner walls of the fluid bed coater or Würster insert and dry, or which dry before impinging on the beads. The second possibility to explain the presence of PVP is that it could migrate through the controlled release layer to the surface of the bead. Prior to drying of the sprayed polymer in the processor, the wetted particle could give rise to enhanced polymer mobility, leading to migration. It must be noted that while there is a clear presence of the PVP marker ion, very limited marker ion intensity of INDO was observed in the outer layer (only in ERL 100\%, ERL 100\% latex and EC surfaces). As the sprayed solution contains both INDO and PVP, there is no reason why INDO should not be present on the coater wall or should not migrate through the ERL layer together with the PVP. Firstly the specific ion for INDO has a much higher mass compared to the PVP marker ion, making it more specific but less intense. Secondly, the glass solution only contains $30 \%$ (w/w) of INDO opposite to $70 \%$ (w/w) PVP. These two reasons could account for the smaller INDO marker intensities observed on ERL and EC surfaces. 
When PVP is added as a pore former, it is more evenly distributed on the outer surface compared to the spots observed in the pure ERL or EC surfaces. For ERL based surfaces, a higher PVP marker intensity was observed in coatings containing $25 \%$ of PVP compared to those containing only $10 \%$ of pore former (Figures 5). The opposite was observed in EC based surfaces (Figure 7), but here, the high variability of the PVP marker intensities point to high variability in surface distribution and constitution. This could also be concluded when taking into account the distribution of PVP along the surface. Whereas in the ERL coated samples, the PVP is more evenly spread and present over the entire surface of the coated beads (Fig. 4b,c Column 3), in the EC coated samples a more localized PVP presence could be observed (Fig. 6b,c Column 3). This further points to more heterogeneity of EC-PVP surfaces compared to ERL-PVP surfaces.

\section{Composition of coated bead cross-sections}

Although an additional component was introduced in the coated bead cross-sections, the sucrose starting core, it was still possible to distinguish all compounds, i.e. finding marker ions for all chemical entities despite very similar structural compositions (both sucrose and EC are complex carbohydrates). All investigated samples show very distinct layering, namely a glass solution layer on top of the sugar core and an ERL or EC based layer on top of the glass solution. Consecutively coating both layers, even from an identical solvent did not result in some kind of transition layer between both coatings.

The presence of the PVP ion marker in pure ERL layers is not as pronounced as it was with the surface analysis, as only a diffuse presence along the ERL 100\% latex layer is observed. When PVP is used as a pore former, there is an even distribution along the top coating layers. In pure EC layers, the diffuse presence of PVP can be attributed to the properties of the control sample. Again, when PVP is added as a pore former, the PVP marker intensity is significantly more pronounced along the top coating layer.

Analyzing cross sections allowed for the investigation of potential INDO and/or PVP migration into the top coating ERL or EC layer. An inner, middle and outer layer was created for all samples by defining different regions of interest. The ERL based coating showed decreasing PVP and INDO marker intensities along the top coating membrane, irrespective of the membrane constitution. This intensity gradient was higher for INDO marker intensities as opposed to PVP marker intensities, pointing to an increased INDO presence in the inner layer of the top coating membrane. This was most pronounced in ERL 100\% latex membrane. 
Because this membrane is coated from an aqueous dispersion, the water used as a spraying vehicle, is not removed from the system as easily as ethanol, despite the higher coating chamber temperature $\left(60^{\circ} \mathrm{C}\right.$ versus $\left.50^{\circ} \mathrm{C}\right)$. This in turn can enhance molecular mobility of INDO and cause a migration into this outer membrane. Another explanation can be the curing step of the latex formulation where beads are stored at higher temperature compared to the beads coated from an ethanol solution. Similar intensity gradients are observed at EC top coated membranes, although less pronounced. EC-PVP $75-25 \%$ is the only membrane not showing this gradient. The reason for this is unknown. It has to be remarked that the migration of INDO and PVP is very limited along the top coating membranes. INDO for example, has an intensity of maximum $15 \%$ in the entire EC or ERL top coatings compared to glass solution marker intensity. This limited migration of INDO and PVP cannot explain the presence of PVP spots, observed along the pure ERL or EC surfaces. Because there isn't a clear distributional evidence for this in the secondary ion images (no or very small and homogeneous marker presence), it can be concluded that these PVP spots at the surface are a result of contaminations during coating (collisions with dried powder particles on the coating walls). When small enough, this could generate spots on the surface which are not visible at a cross sections of the outer mebranes.

\section{Polymer miscibility}

The mDSC study of miscibility between the rate controlling polymers EC or ERL on one hand and the pore former PVP on the other hand, shows a different phase behavior of these polymer mixtures. Neither EC-PVP nor ERL-PVP completely mix, which is apparent by the presence of two Tg's. Both glass transition temperatures stay fairly constant in case of the EC-PVP blends, which indicates immiscibility between both components. In case of the ERL-PVP blends we can observe an increase in the Tg of PVP with increasing PVP content. This shows that both polymers seem to be at least partially miscible. The decrease in Tg of ERL with increasing PVP content may be due to the fact that PVP is hygroscopic and will have a higher amount of residual sorbed water and most likely also ethanol content. The poor miscibility of both polymer blends corresponds with previously made observations with ToF-SIMS, which showed separate PVP domains in EC or ERL based layers. The partial miscibility between ERL and PVP can also explain why the ERL top coating is better attached to the glass solution layer. This is in contrast with the EC coating, which shows complete immiscibility with PVP, which is, at the most, only loosely connected. This immiscibility between EC and PVP can also be the explanation for the more localized PVP domains, when PVP is used as a pore former in EC coatings, as observed in ToF-SIMS surface images. 


\section{Glass solution phase analysis}

It has been previously shown that an INDO-PVP 30-70\% (w/w) coating surrounding a sucrose pellet forms a glass solution, i.e. a molecularly dispersed drug in a polymeric carrier, resulting in a one phase system ${ }^{12}$. It remained, however, possible that the application of a rate controlling membrane on top of this glass solution could potentially cause instability to this glass solution through crystallization of indomethacin. This could be especially true when the rate controlling membrane was applied from an aqueous dispersion, because it is well known that the presence of water, even in the form of water vapor, can potentially result in drug crystallization. From the results of the XRPD analysis of the coated beads it became apparent that this is not the case. As the measurements were performed in transmission mode, all different layers of the beads were scanned which is evidenced by the presence of the characteristic Bragg peaks of the sucrose pellets in all diffractograms. However, no characteristic Bragg peaks of crystalline indomethacin were present in any of the samples, indicating that indomethacin remains X-ray amorphous after the application of a rate controlling membrane, whether this is from an ethanol solution or an aqueous dispersion. 


\section{Conclusions}

In this study, the composition and polymer distribution of complex coated systems was elucidated by combining complementary solid state analytical techniques. Sucrose cores were coated with an INDO-PVP glass solution layer and on top of this, a rate controlling membrane was applied. These membranes consisted of EC or ERL with or without a pore former (PVP), coated from a solution or an aqueous dispersion, and with or without plasticizer (TEC) added.

SEM images revealed vastly different topography of the surface of the different coated beads with a cracked surface for INDO-PVP glass solution, a rough surface for ERL coating from a solution and smooth surface for ERL coated from a dispersion. This difference can be appointed to the different film formation process and an additional curing step for the dispersion. Cross sections revealed a difference in structure between ERL and EC based layers being smooth and well attached to the glass solution substrate or presenting a more layered structure loosely attached to substrate respectively. The difference in attachment can be attributed to the fact that ERL is partially miscible with PVP and EC is immiscible.

ToF-SIMS analysis showed to be an excellent method to provide details regarding the chemical composition of the surfaces and the distribution of the different components. The ToF-SIMS analysis showed the unexpected presence of PVP in layers made up of pure ERL or EC, originating from residual presence in the fluid bed coater. INDO and PVP show very limited migration into the outer membranes. Furthermore, there is a different localization in the samples containing PVP as a pore former. In the ERL-PVP coatings, the PVP seems to be more evenly distributed throughout the surface, whereas in the EC-PVP coatings, the presence of PVP seems to be more localized. This difference can be explained by the miscibility differences shown in mDSC and can have a vast effect on drug diffusion through this layer.

XRPD analysis revealed that the application of a rate controlling membrane does not affect the solid state properties of the underlying glass solution, even when coated from an aqueous dispersion.

This study reveals a unique insight into complex coated systems (glass solution + controlled release layer) on inert carriers. Rate controlling polymer selection, pore former selection and concentration can all have major consequences for the resulting phase behavior, and deposition onto the carrier. This in turn will have a vast impact on the performance of this type of drug delivery system. 


\section{References}

1. Ayenew Z, Paudel A, Van den Mooter G 2012. Can compression induce demixing in amorphous solid dispersions? A case study of naproxen-PVP K25. Eur J Pharm Biopharm 81: 207-213.

2. Varum FJO, Merchant HA, Basit AW 2010. Oral modified-release formulations in motion: The relationship between gastrointestinal transit and drug absorption. Int $\mathbf{J}$ Pharm 395: 26-36.

3. Garbacz G, Klein S 2012. Dissolution testing of oral modified-release dosage forms. J Pharm Pharmacol 64: 944-968.

4. Lopez FL, Ernest TB, Tuleu C, Gul MO 2015. Formulation approaches to pediatric oral drug delivery: benefits and limitations of current platforms. Expert Opin Drug Deliv 12: 1727-1740.

5. Yang Z, Nollenberger K, Albers J, Craig D, Qi S 2015. Molecular Indicators of Surface and Bulk Instability of Hot Melt Extruded Amorphous Solid Dispersions. Pharm Res 32: $1210-1228$.

6. Tran PHL, Tran TTD, Park JB, Lee BJ 2011. Controlled release systems containing solid dispersions: strategies and mechanisms. Pharm Res 28: 2353-2378.

7. Baird JA, Taylor LS 2012. Evaluation of Amorphous Solid Dispersion Properties Using Thermal Analysis Techniques. Adv Drug Deliv Rev 64: 396-421.

8. Newman A, Engers D, Bates S, Ivanisevic I, Kelly RC, Zografi G 2008. Characterization of amorphous API : Polymer mixtures using X-ray powder diffraction. J Pharm Sci 97: 4840-4856. 
9. Yun Y, Zou X, Hovmöller S, Wan W 2015. Three-dimensional electron diffraction as a complementary technique to powder X-ray diffraction for phase identification and structure solution of powders. IUCrJ 2: 267-282.

10. Liu J, Saw RE, Kiang YH 2010. Calculation of effective penetration depth in X-ray diffraction for pharmaceutical solids. J Pharm Sci 99: 3807-3814.

11. Thakral NK, Yamada H, Stephenson GA, Suryanarayanan R 2015. Spatial Distribution of Trehalose Dihydrate Crystallization in Tablets by X-ray Diffractometry. Mol Pharm 12: $3766-3775$.

12. Dereymaker A, Van den Mooter G 2015. The Peculiar Behavior of the Glass Transition Temperature of Amorphous Drug-Polymer Films Coated on Inert Sugar Spheres. J Pharm Sci 104: 1759-1766.

13. Fletcher JS, Rabbani S, Henderson A, Blenkinsopp P, Thompson SP, Lockyer NP, Vickerman JC 2008. A New Dynamic in Mass Spectral Imaging of Single Biological Cells. Anal Chem 80: 9058-9064

14. Touboul D, Halgand F, Brunelle A, Kersting R, Tallarek E, Hagenhoff B, Laprévote O 2004. Tissue Molecular Ion Imaging by Gold Cluster Ion Bombardment. Anal Chem 76: $1550-1559$

15. Kraft ML, Klitzing HA 2014. Imaging lipids with secondary ion mass spectrometry Biochimica et Biophysica Acta 1841: 1108-1119

16. Passarelli MK, Winograd N 2014. Lipid imaging with time-of-flight secondary ion mass spectrometry (ToF-SIMS) Biochimica et Biophysica Acta 1811: 976-990 
17. Bodzon-Kulakowska A, Suder P 2016. Imaging mass spectrometry: Instrumentation, applications, and combination with other visualization techniques. Mass Spectrometry Reviews 35: 147-169

18. McPhail DS 2006. Applications of Secondary Ion Mass Spectrometry (SIMS) in Materials Science. J Mater Sci 41: 873-903

19. Belu AM, Davies MC, Newton JM, Patel N 2000. ToF-SIMS characterization and imaging of controlled-release drug delivery systems. Anal Chem 72: 5625-5638.

20. Barnes TJ, Kempson IM, Prestidge CA 2011. Surface analysis for compositional, chemical and structural imaging in pharmaceutics with mass spectrometry: a ToF-SIMS perspective. Int J Pharm 417: 61-69.

21. Meeus J, Chen X, Scurr DJ, Ciarnelli V, Amssoms K, Roberts CJ, Davies MC, Van den Mooter G 2012. Nanoscale surface characterization and miscibility study of a spraydried injectable polymeric matrix consisting of poly(lactic-co-glycolic acid) and polyvinylpyrrolidone. J Pharm Sci 101: 3473-3485.

22. Meeus J, Scurr DJ, Chen X, Amssoms K, Davies MC, Roberts CJ, Van den Mooter G 2015. Combination of (M)DSC and Surface Analysis to Study the Phase Behaviour and Drug Distribution of Ternary Solid Dispersions. Pharm Res 32: 1407-1416

23. Meeus J, Lenaerts M, Scurr DJ, Amssoms K, Davies MC, Roberts CJ, Van den Mooter G 2015. The Influence of Spray-Drying Parameters on Phase Behavior, Drug Distribution, and In Vitro Release of Injectable Microspheres for Sustained Release. J Pharm Sci 104: 1451-1460

24. Meeus J, Scurr DJ, Appeltans B, Amssoms K, Annaert P, Davies MC, Roberts CJ, Van den Mooter G 2015. Influence of formulation composition and process on the 
characteristics and in vitro release from PLGA-based sustained release injectables. Eur J Pharm Biopharm 90:22-29

25. Brown A, Vickerman JC 1984. Static SIMS for applied surface analysis. Surf Interface Anal. 6: 1-14.

26. Jesson DA, Watts JF 2012. The Interface and Interphase in Polymer Matrix Composites: Effect on Mechanical Properties and Methods for Identification. Polym Rev 52: 321 354.

27. Lietz CB, Gemperline E, Li L 2013. Qualitative and quantitative mass spectrometry imaging of drugs and metabolites. Adv Drug Deliv Rev 65: 1074-1085.

28. Prestidge CA, Barnes TJ, Skinner W 2007. Time-of-flight secondary-ion mass spectrometry for the surface characterization of solid-state pharmaceuticals. J Pharm Pharmacol 59: 251-259 
Graphical abstract

Fluid bed coating
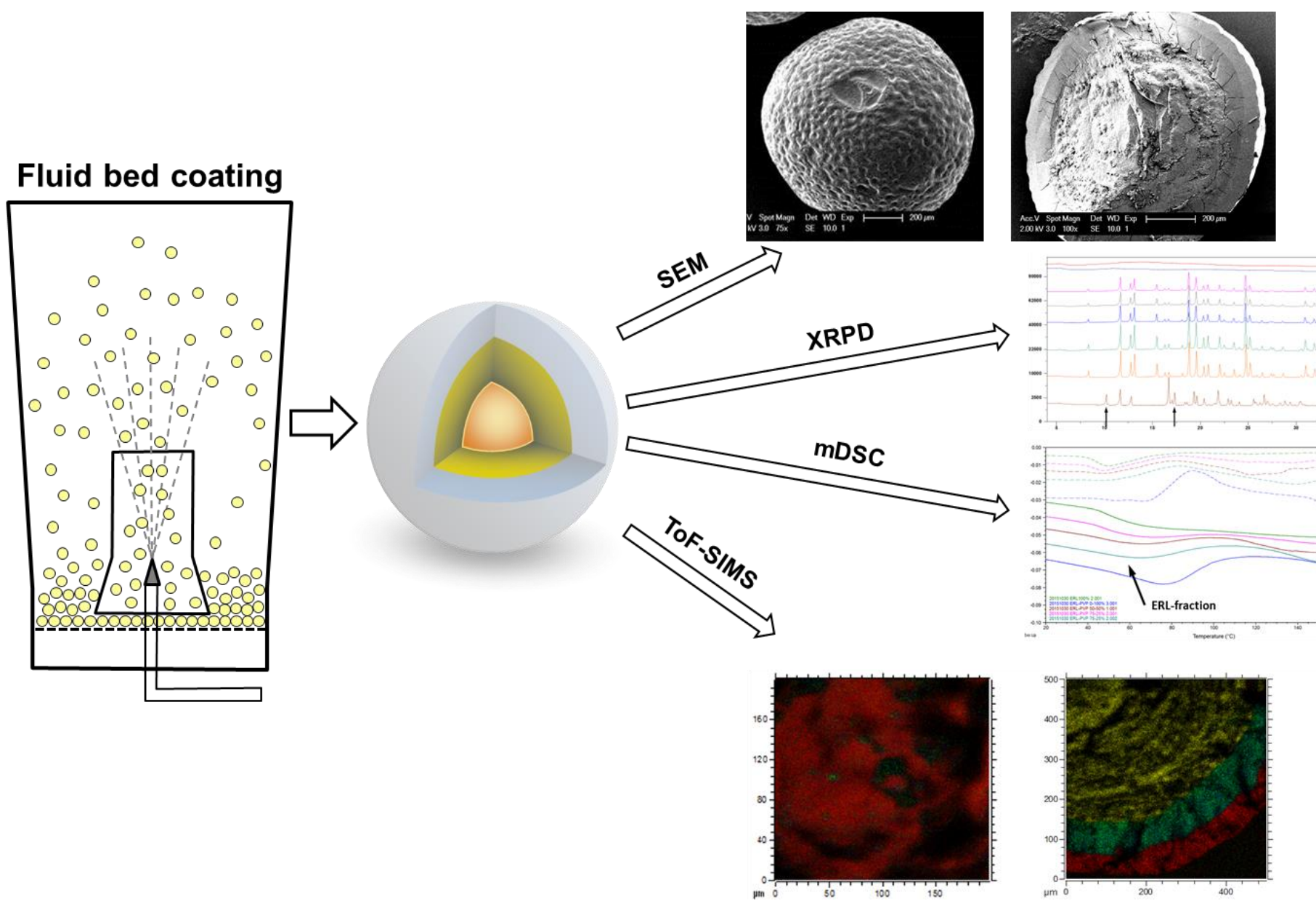


\section{Figures}

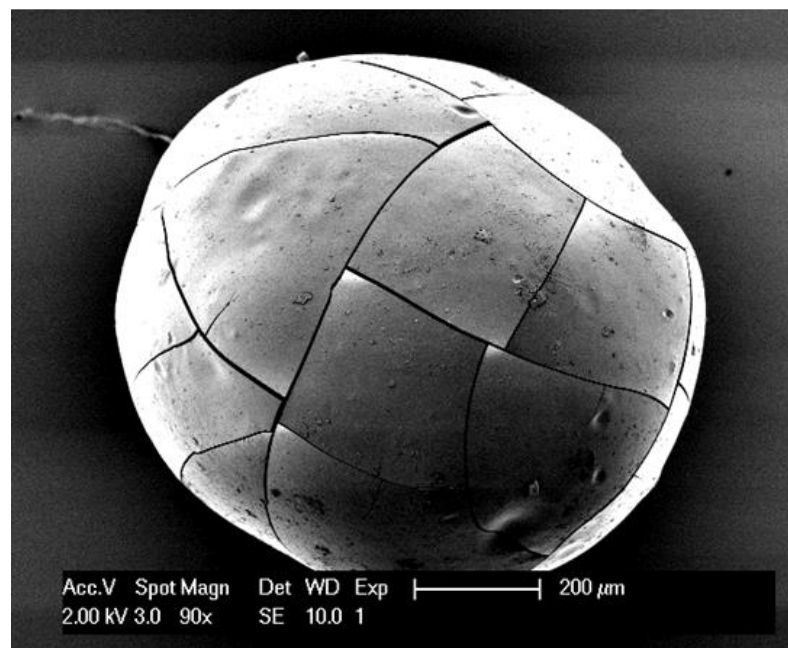

A

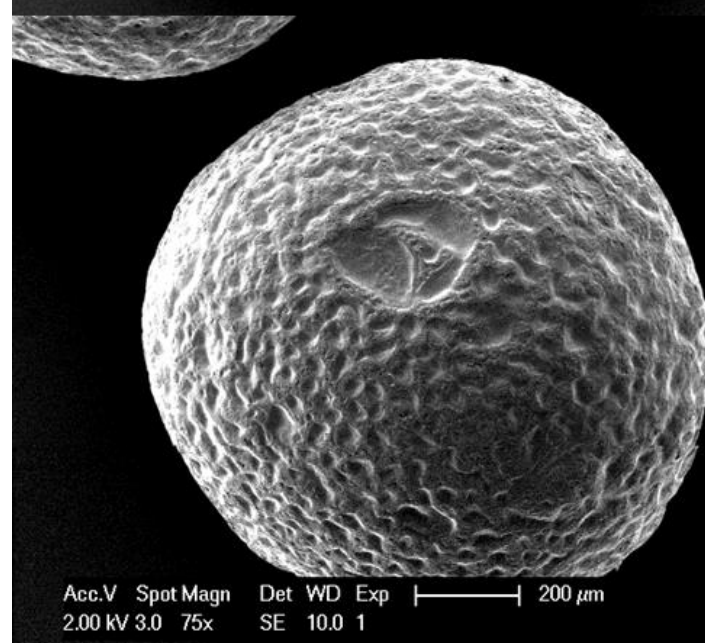

\section{C}
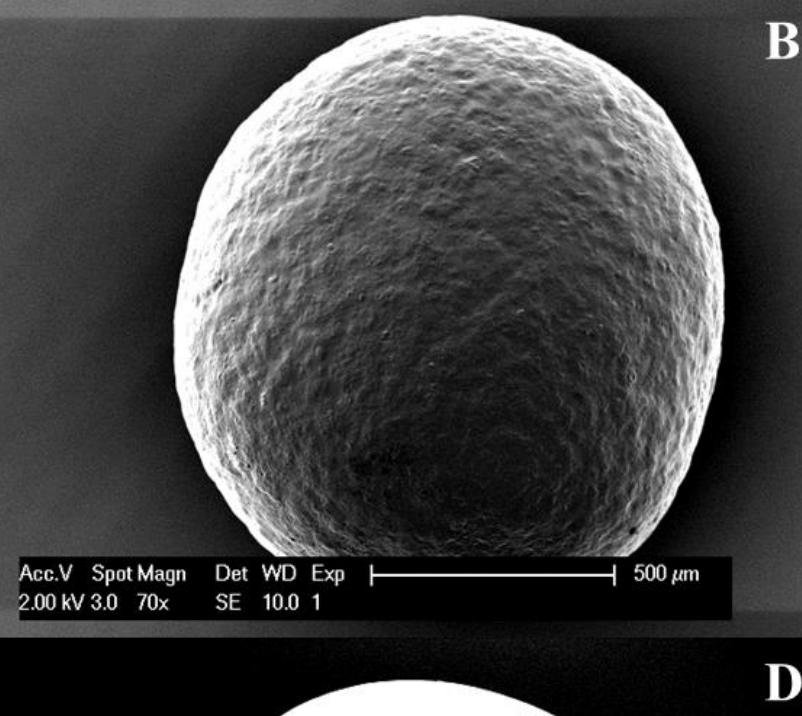

Figure 1: SEM micrographs of INDO-PVP coated glass solution bead (A), EC top coated bead (B), ERL to bead from an ethanolic solution (C) and ERL top coated bead from an aqueous dispersion (D) 


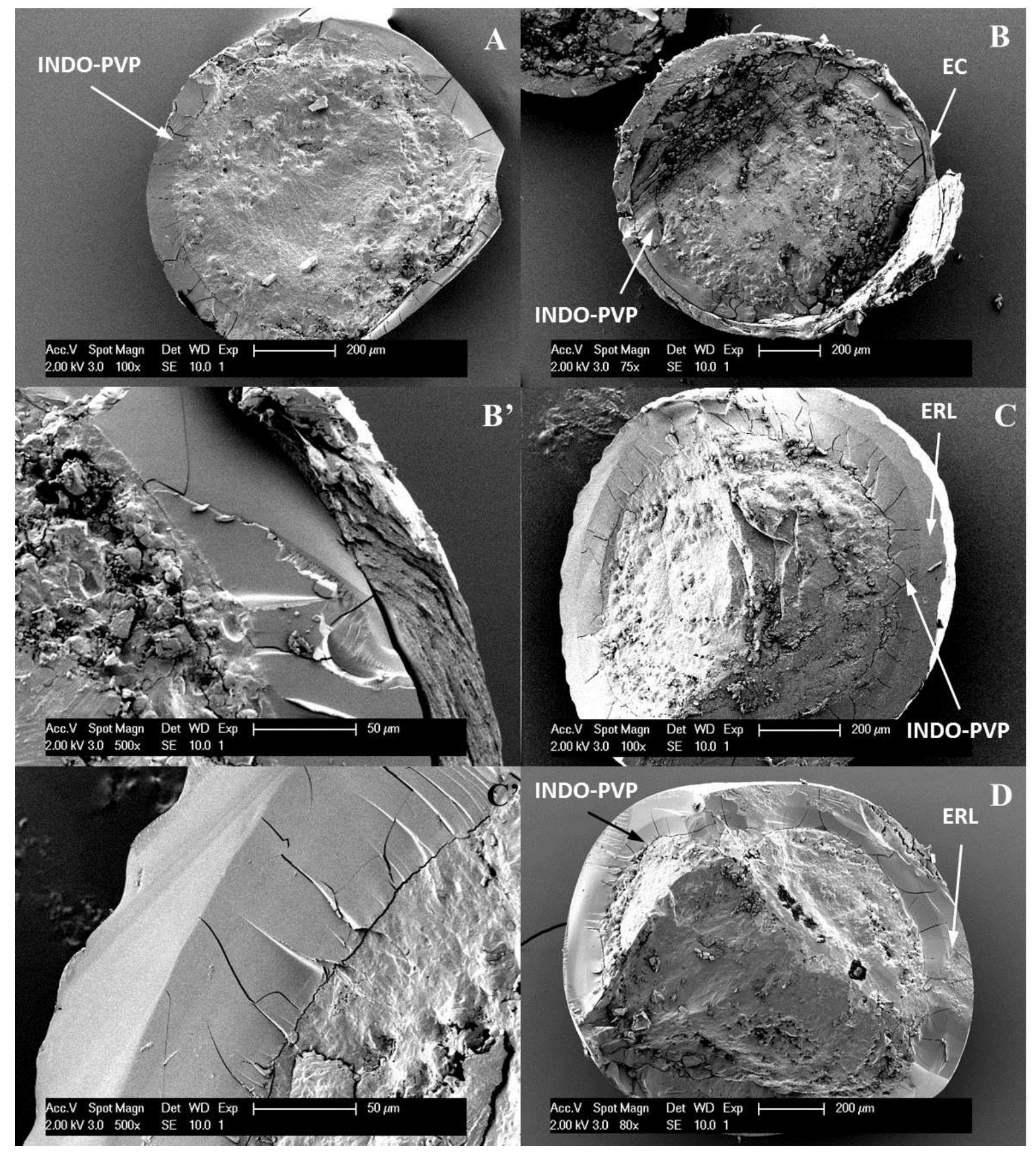

Figure 2: SEM micrographs of INDO-PVP coated glass solution bead (A), EC top coated bead (B) and a detaile on the coating layers (B'), ERL top coated bead from an ethanolic solution (C) with a detailed view on the layers (C'), and, an ERL top coated bead from an aqueous dispersion (D). 

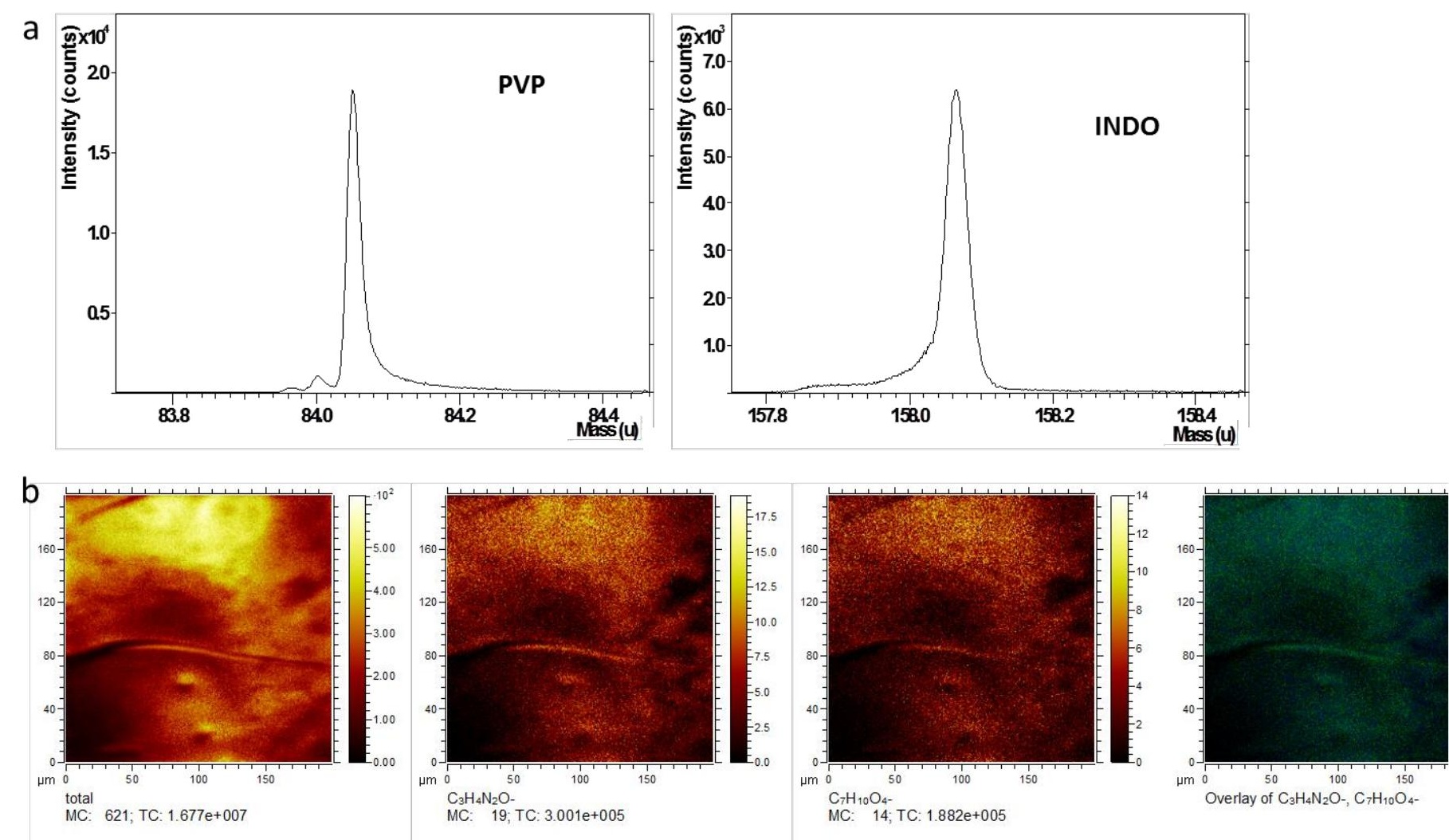

Figure 3: ToF-SIMS ion spectra of INDO-PVP coated glass solution (a) at PVP and INDO marker positions. T, SIMS ion images of INDO-PVP coated glass solution (b) with the total ion image, the PVP marker image, the IN] marker image and an overlay of PVP marker image (green) and INDO marker image (blue). 


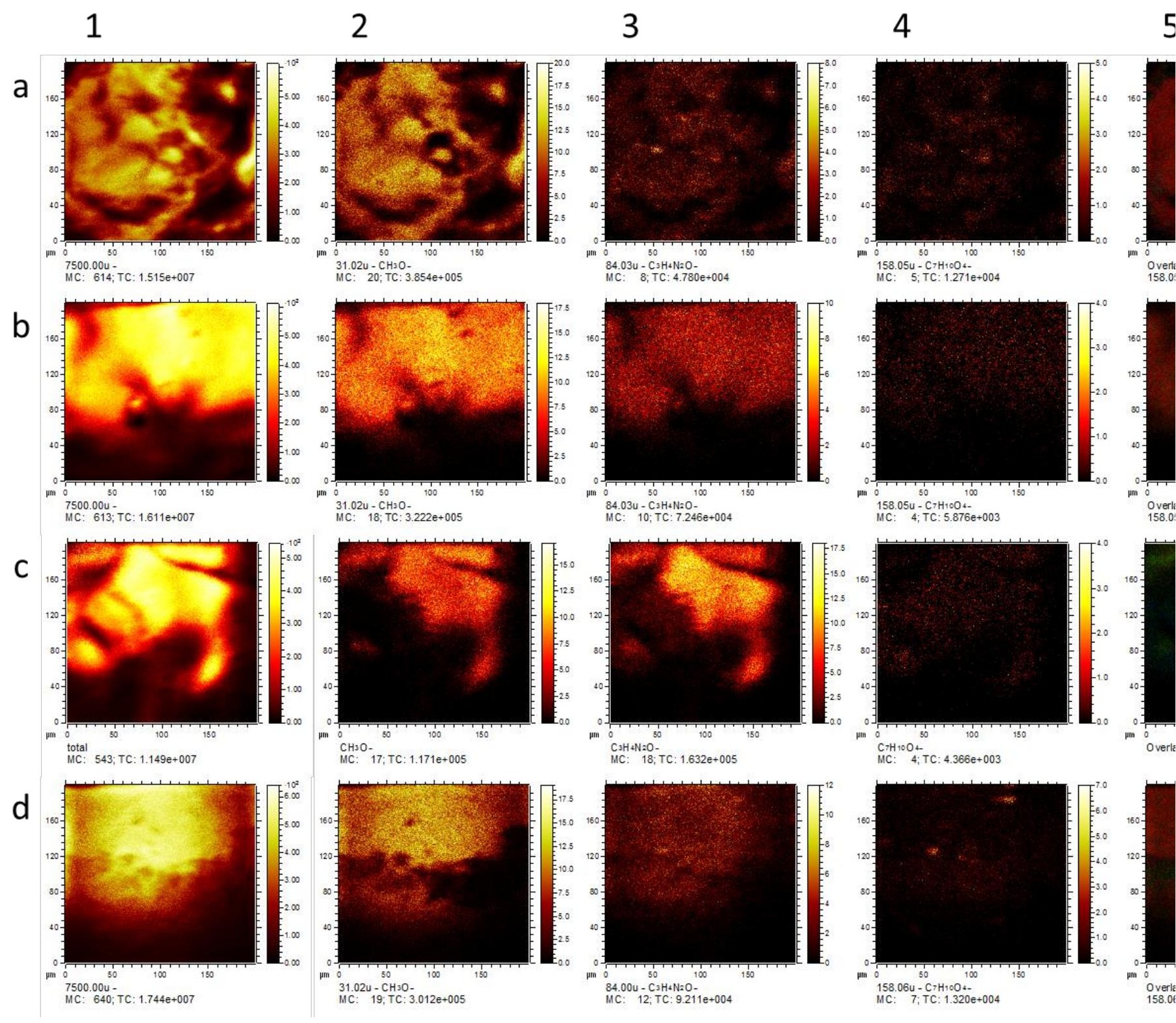

Figure 4: ToF-SIMS ion images of ERL coated formulations, 100\% ERL (row a), ERL-PVP 90-10\% (row b), ERL-PVP 75-25\% (row c) and 100\% ERL latex (row d). Column 1 represents the total ion images o all formulations, column 2 the ERL marker images, column 3 the PVP marker images, column 4 the INDC marker images and column 5 an overlay image of ERL marker (red), PVP marker (green) and INDC marker (blue). 


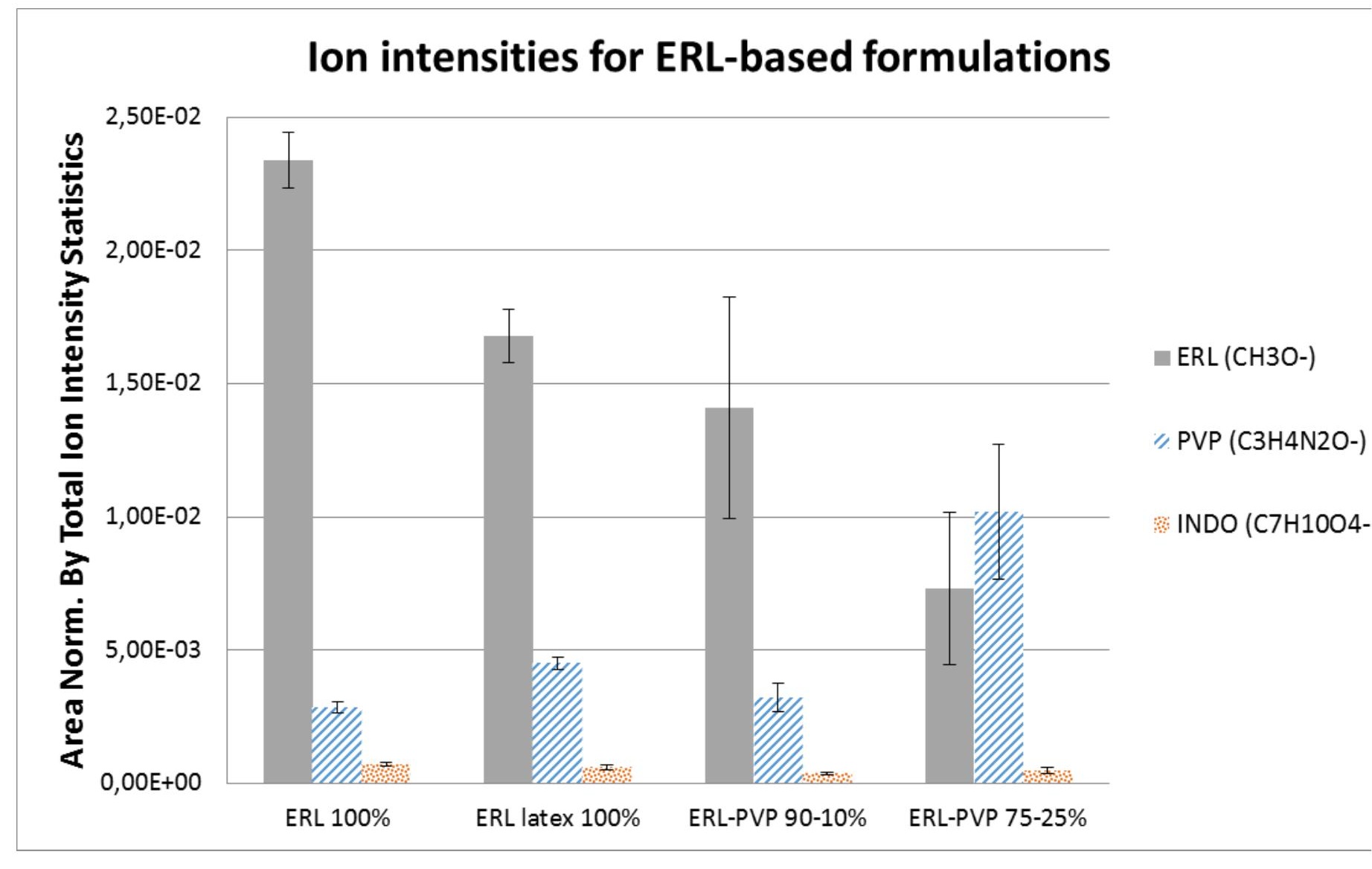

Figure 5: Ion intensities for the different formulations containing an ERL-based outer coating layer. ERL marke intensities are represented in filled grey bars, PVP marker intensities are striped blue bars and INDO marke intensities are orange dotted bars. Ion intensities are measured as a mean of 3 surfaces and the area is normalizec by total ion statistics. 


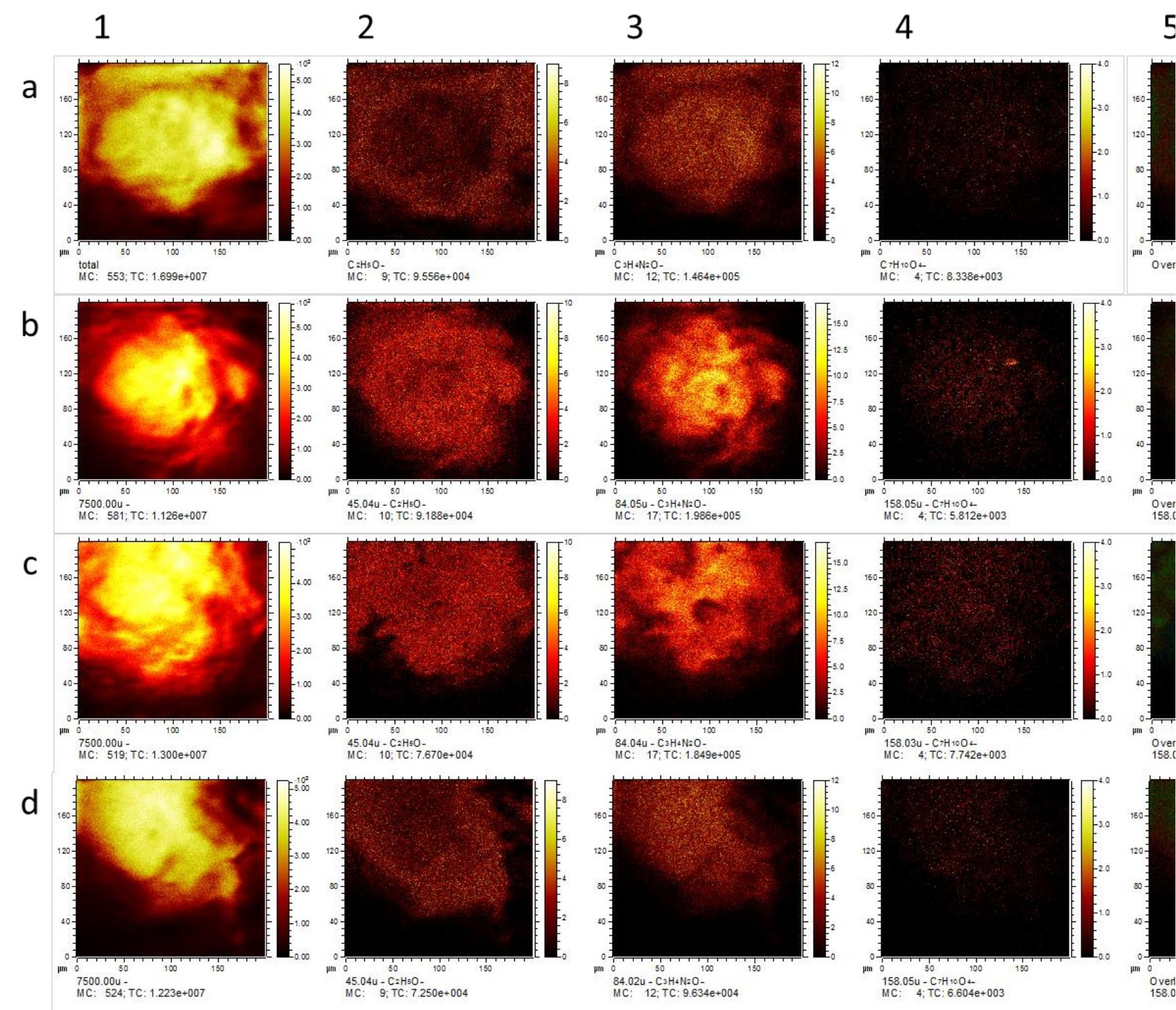

Figure 6: ToF-SIMS ion images of EC coated formulations, 100\% EC (row a), EC-PVP 90-10\% (row b) (row c) and EC 100\% - TEC (row d). Column 1 represents the total ion images of all formulations, colum images, column 3 the PVP marker images, column 4 the INDO marker images and column 5 an overlay in (red), PVP marker (green) and INDO marker (blue). 


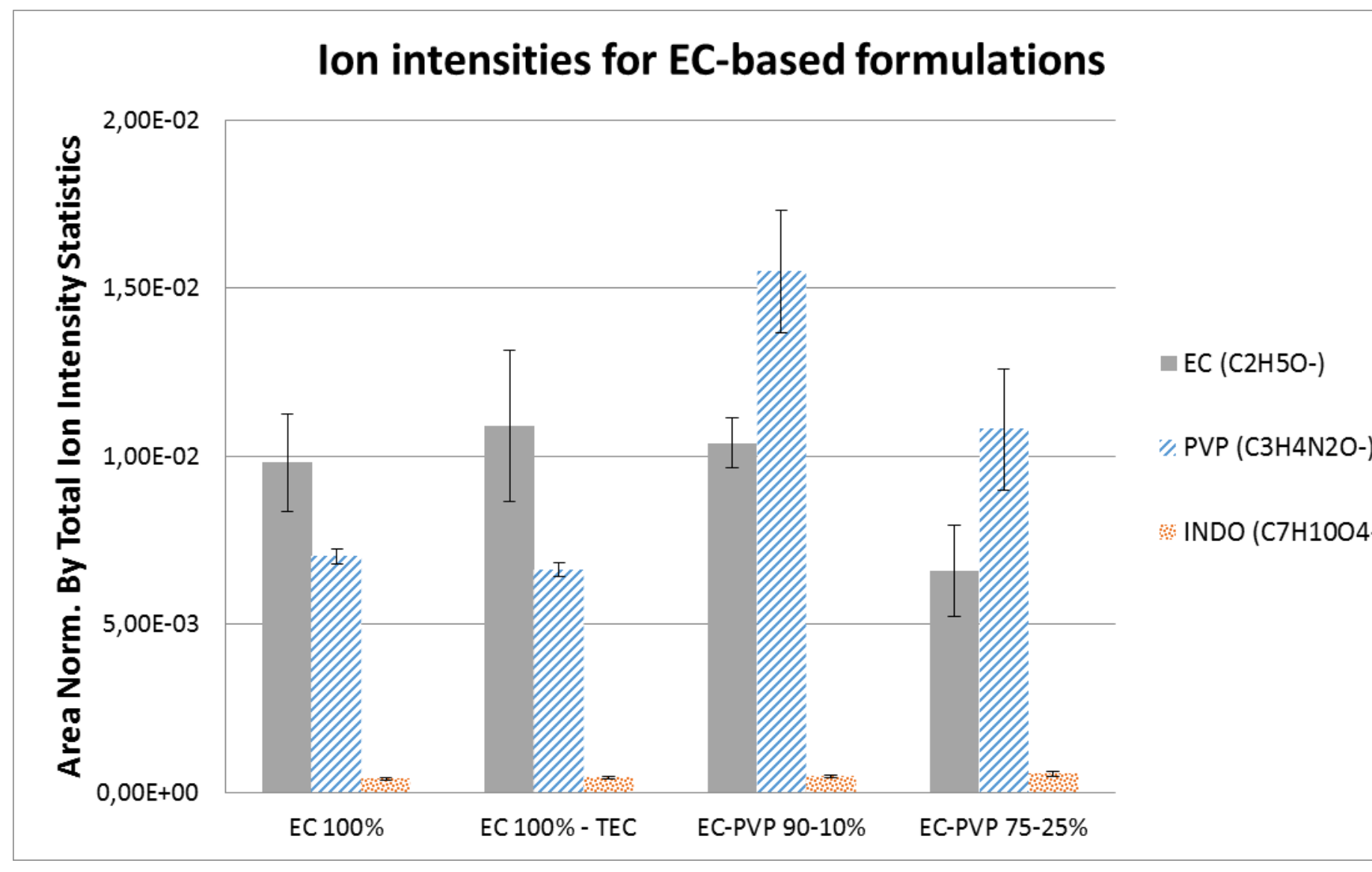

Figure 7: Ion intensities for the different formulations containing an EC-based outer coating layer. EC mar intensities are represented in filled grey bars, PVP marker intensities are striped blue bars and INDO mar intensities are orange dotted bars. Ion intensities are measured as a mean of 3 surfaces and the area is normaliz by total ion statistics.

1

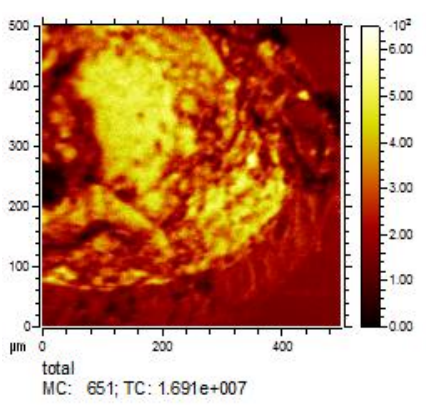

2

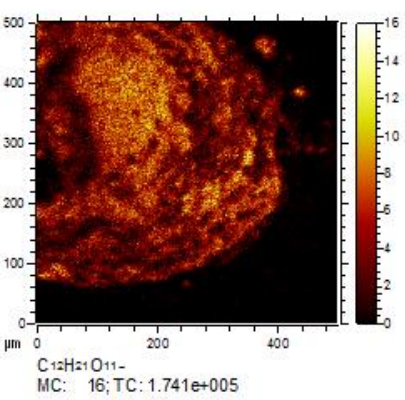

3

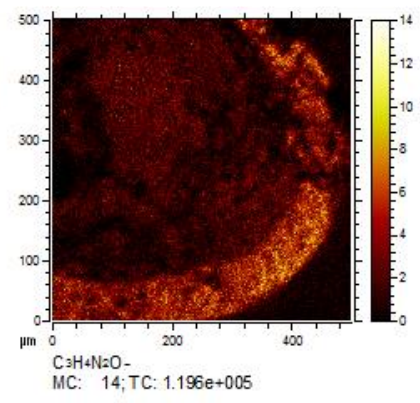

4

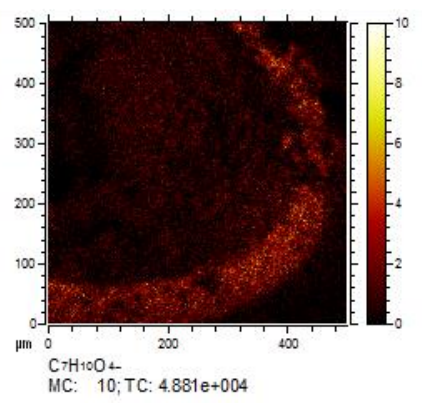

Figure 8: ToF-SIMS ion images of a cross-section of an INDO-PVP glass solution coated bead. Column 1 represents the total ion images of all formulations, column 2 the sucrose marker images, column 3 the PVP marker images, column 4 the INDO marker images and column 5 an overlay image of sucrose marker (red), PVP marker (green) and INDO marker (blue). 

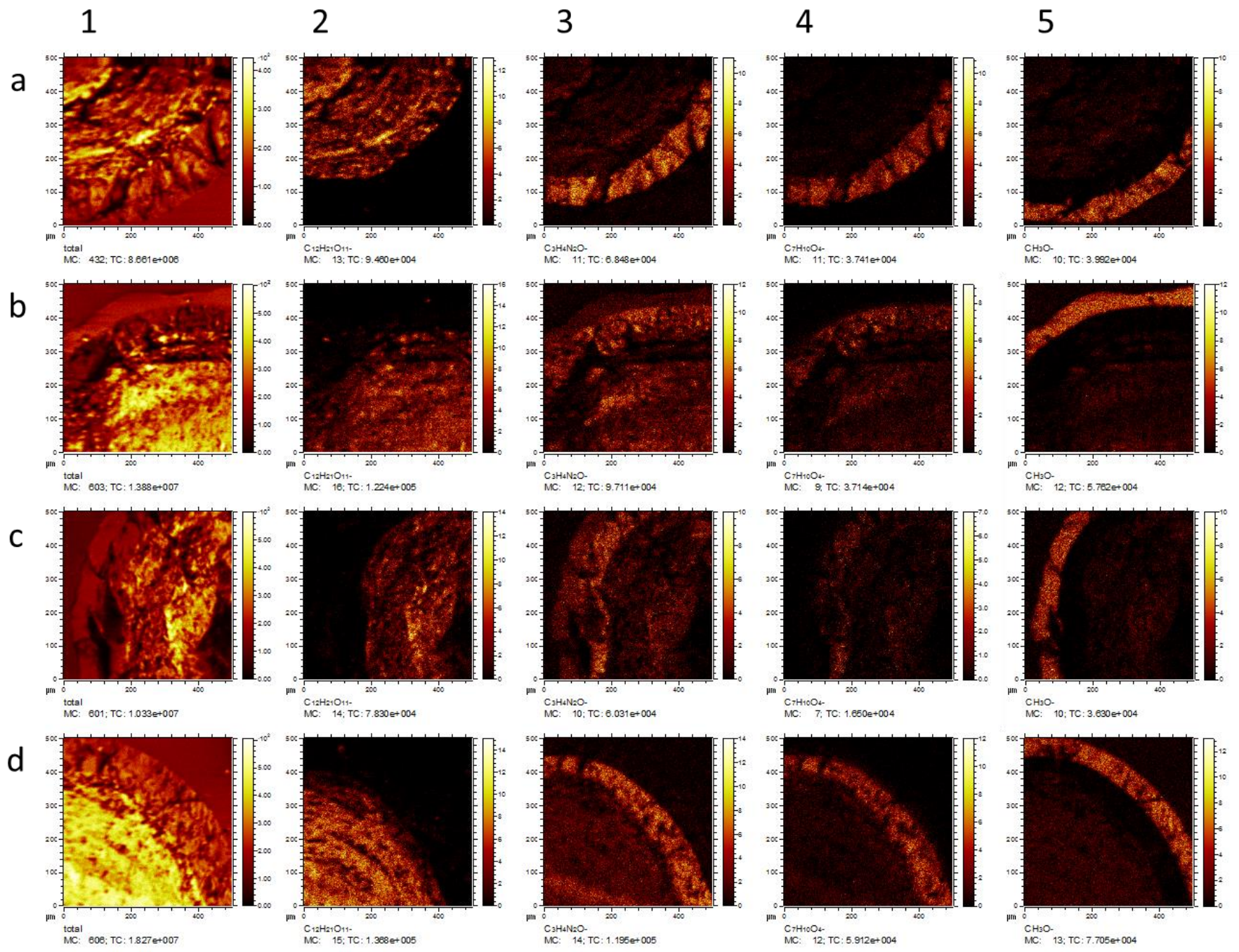

Figure 9: ToF-SIMS ion images of the cross sections of ERL top coated formulations, ERL100\% (row a) 90-10\% (row b), ERL-PVP 75-25\% (row c) and ERL 100\% latex (row d). Column 1 represents the total of all formulations, column 2 the sucrose marker images, column 3 the PVP marker images, column 4 marker images, column 5 the ERL marker images and column 6 an overlay image of ERL marker (red), P (green), INDO marker (blue) and sucrose marker (yellow). 


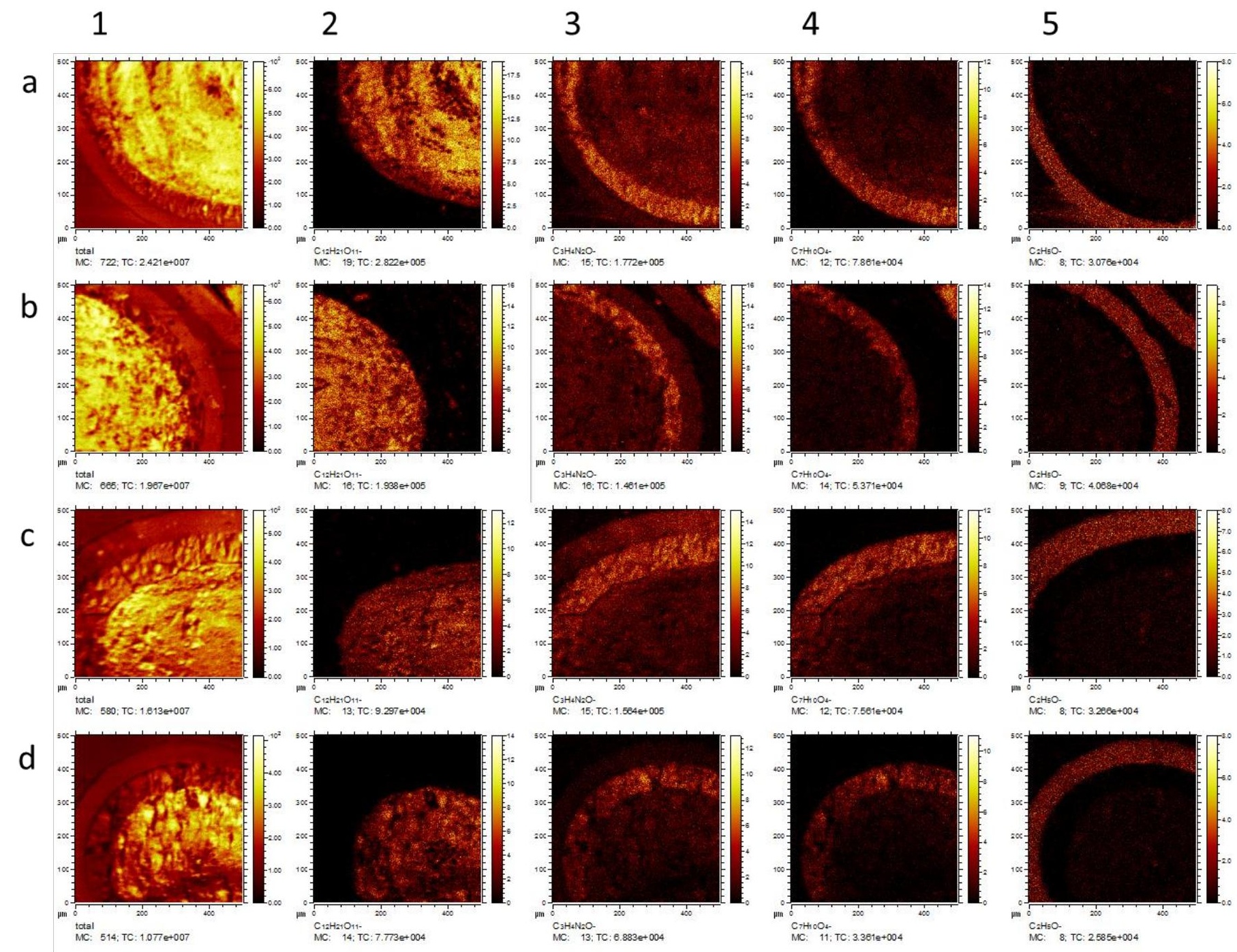

Figure 10: ToF-SIMS ion images of the cross sections of EC top coated formulations, EC 100\% (row a), EC10\% (row b), EC-PVP 75-25\% (row c) and EC 100\% - TEC (row d). Column 1 represents the total ion imas formulations, column 2 the sucrose marker images, column 3 the PVP marker images, column 4 the INDC images, column 5 the ERL marker images and column 6 an overlay image of ERL marker (red), PVP marker INDO marker (blue) and sucrose marker (yellow). 


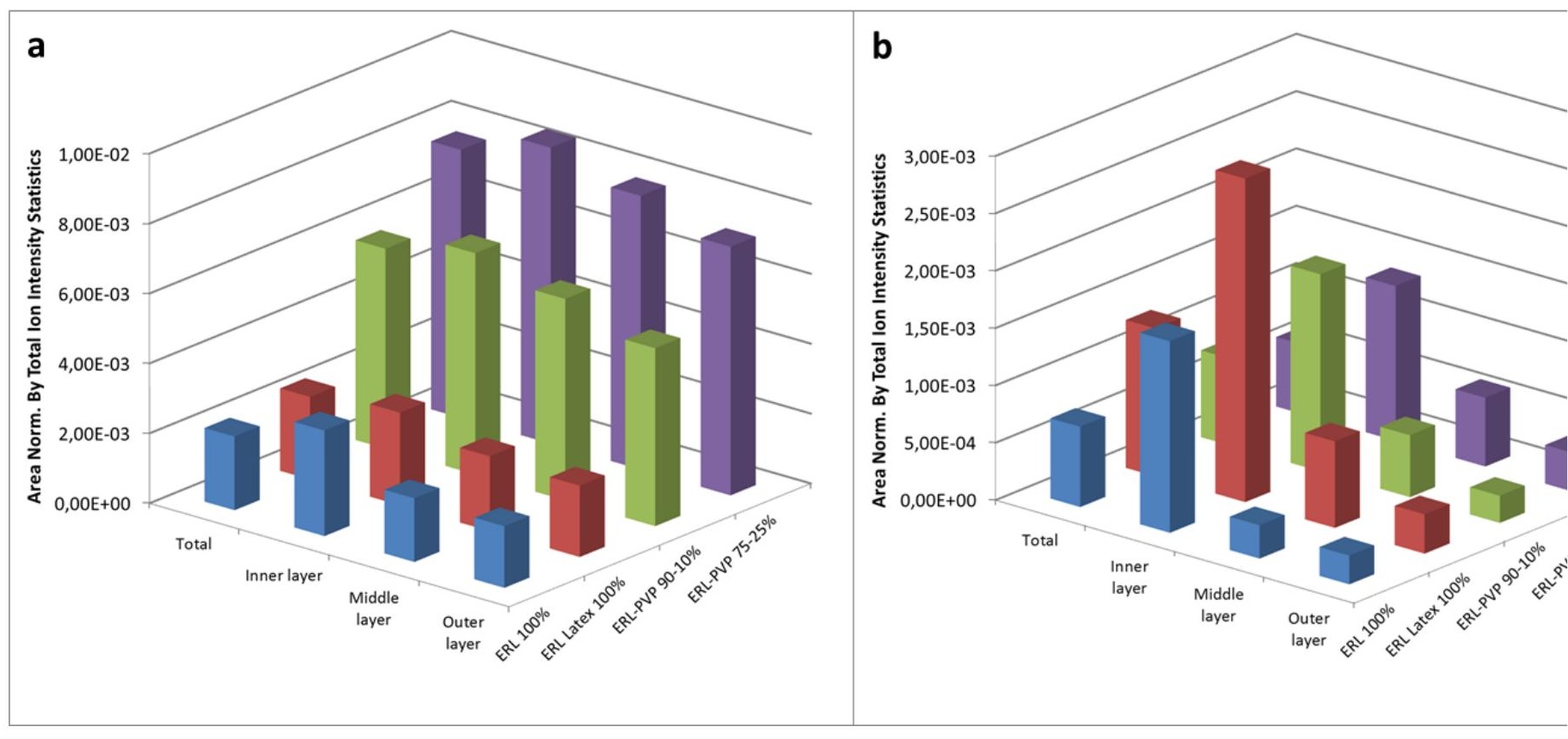

Figure 11: Total, inner layer, middle layer and outer layer intensities of the PVP (a) and INDO (b) ion marke ERL top coatings. ERL 100\% intensities are shown in blue bars, ERL 100\% latex intensities in red bars, E PVP 90-10\% intensities in green bars and ERL-PVP 75-25\% intensities in purple bars.

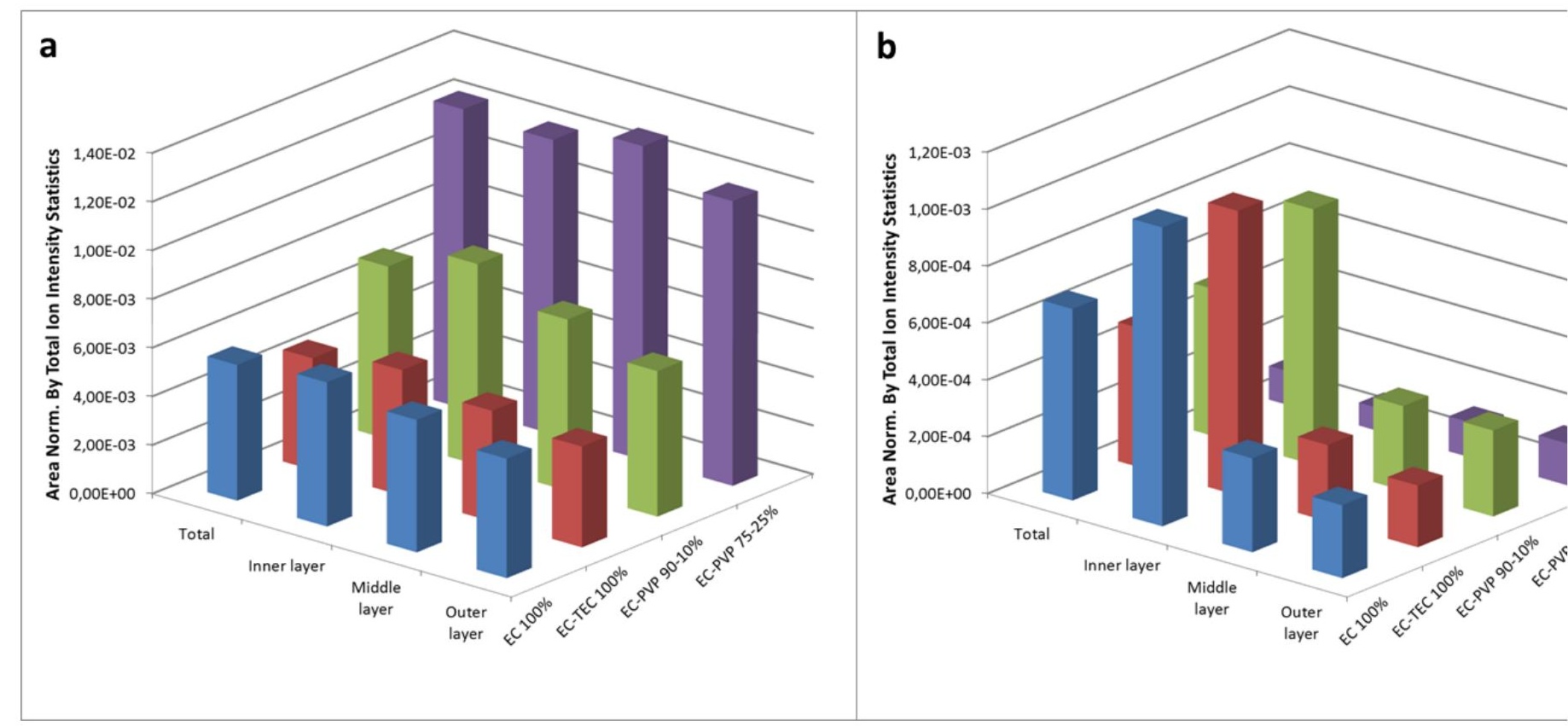

Figure 12: Total, inner layer, middle layer and outer layer intensities of the PVP (a) and INDO (b) ion marke EC top coatings. EC 100\% intensities are shown in blue bars, EC 100\% - TEC intensities in red bars, EC-PVP $10 \%$ intensities in green bars and EC-PVP $75-25 \%$ intensities in purple bars. 
a

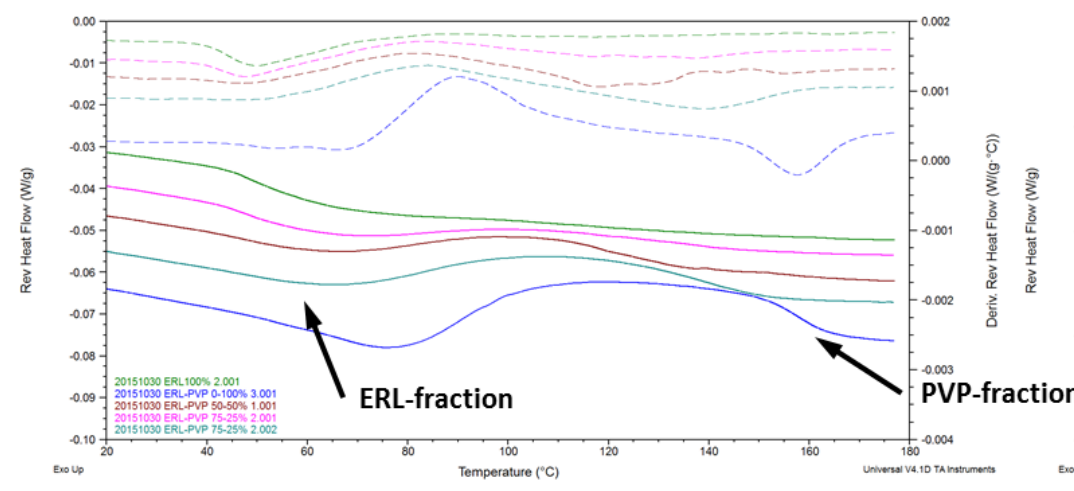

b

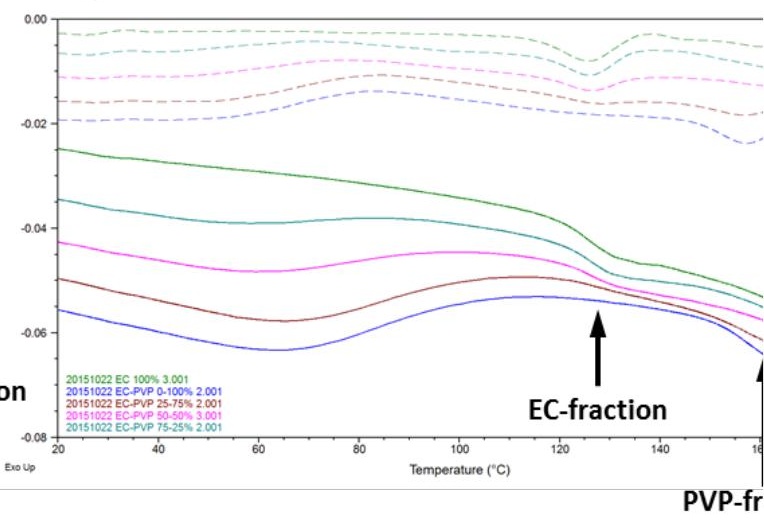

Figure 13: mDSC thermogram overlay of the reversing heat flow (full lines) and the first derivative of the reversing heat flow (dotted lines) of the different spray dried ERL-PVP (a) and EC-PVP (b) polymer mixtures.

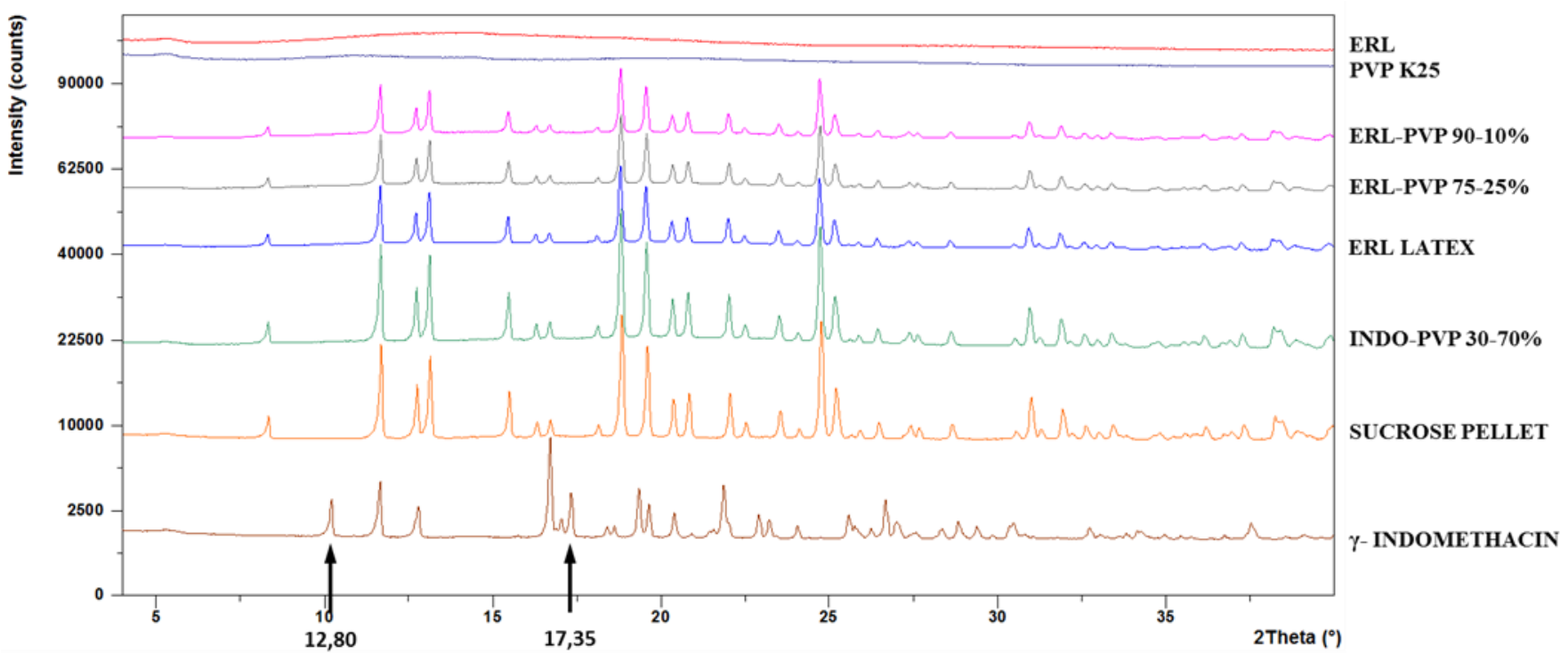

Figure 14: XRPD diffractogram of reference substances (ERL, PVP K25, sucrose pellet and $\gamma$ indomethacin), INDO-PVP glass solution coating and different ERL based coatings (ERL-PVP 90$10 \%$, ERL-PVP $75-25 \%$ and ERL latex coating). Characteristic peaks of $\gamma$-indomethacin are marked with arrows at $12.80^{\circ}$ and $17.35^{\circ}$ 


\section{Tables}

Table 1: Glass transition temperatures of the different spray dried EC-PVP polymer mixtures. All formulations are measured in triplicate with the mean and standard deviation.

\begin{tabular}{|c|c|c|c|c|}
\hline \multirow{2}{*}{ EC/PVP ratio (\%) } & \multicolumn{4}{|c|}{ Glass transition temperature (C') } \\
\cline { 2 - 5 } & \multicolumn{2}{|c|}{ EC-fraction } & PVP-fraction \\
\cline { 2 - 5 } & mean & SD & mean & SD \\
\hline $100 / 0$ & 125,1 & 0,8 & & \\
\hline $75 / 25$ & 125,0 & 0,7 & 161,1 & 1,0 \\
\hline $50 / 50$ & 125,1 & 0,1 & 162,2 & 1,0 \\
\hline $25 / 75$ & 125,5 & 0,6 & 156,9 & 0,4 \\
\hline $0 / 100$ & & & 156,8 & 0,2 \\
\hline
\end{tabular}

Table 2: Glass transition temperatures of the different spray dried ERL-PVP polymer mixtures. All formulations are measured in triplicate with the mean and standard deviation.

\begin{tabular}{|c|c|c|c|c|}
\hline \multirow{2}{*}{ ERL/PVP ratio (\%) } & \multicolumn{4}{|c|}{ Glass transition temperature (C) } \\
\cline { 2 - 5 } & \multicolumn{2}{|c|}{ ERL-fraction } & \multicolumn{2}{c|}{ PVP-fraction } \\
\cline { 2 - 5 } & mean & SD & mean & SD \\
\hline $100 / 0$ & 53,5 & 0,7 & & \\
\hline $75 / 25$ & 52,0 & 1,3 & - & - \\
\hline $50 / 50$ & 50,0 & 1,7 & 123,7 & 2,2 \\
\hline $25 / 75$ & 47,1 & 4,4 & 137,5 & 1,1 \\
\hline $0 / 100$ & & & 156,9 & 0,7 \\
\hline
\end{tabular}




\section{Supplementary Data}
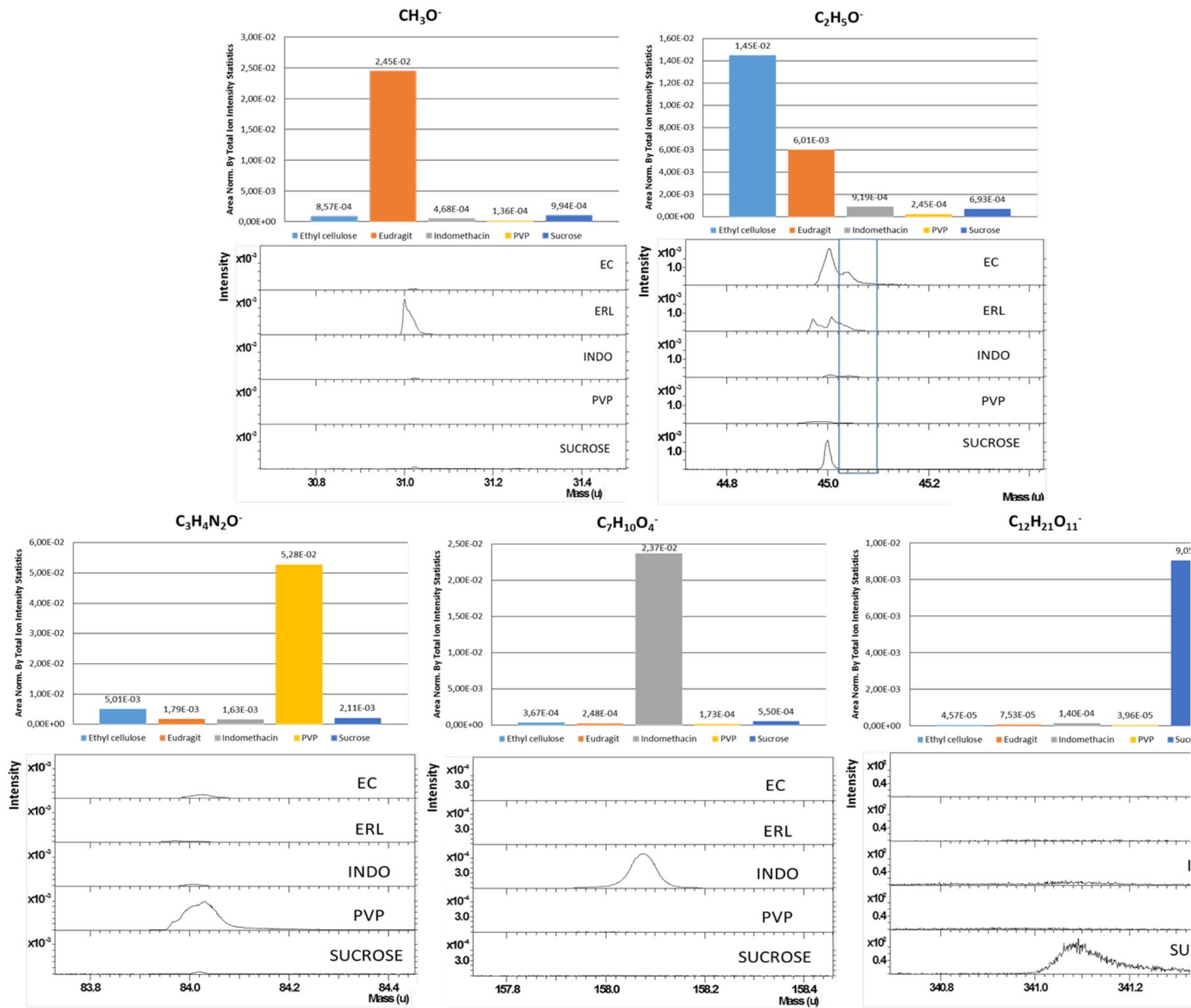

Figure A: Ion intensities and spectra of the component selective ion markers opposed to the other compound controls. 


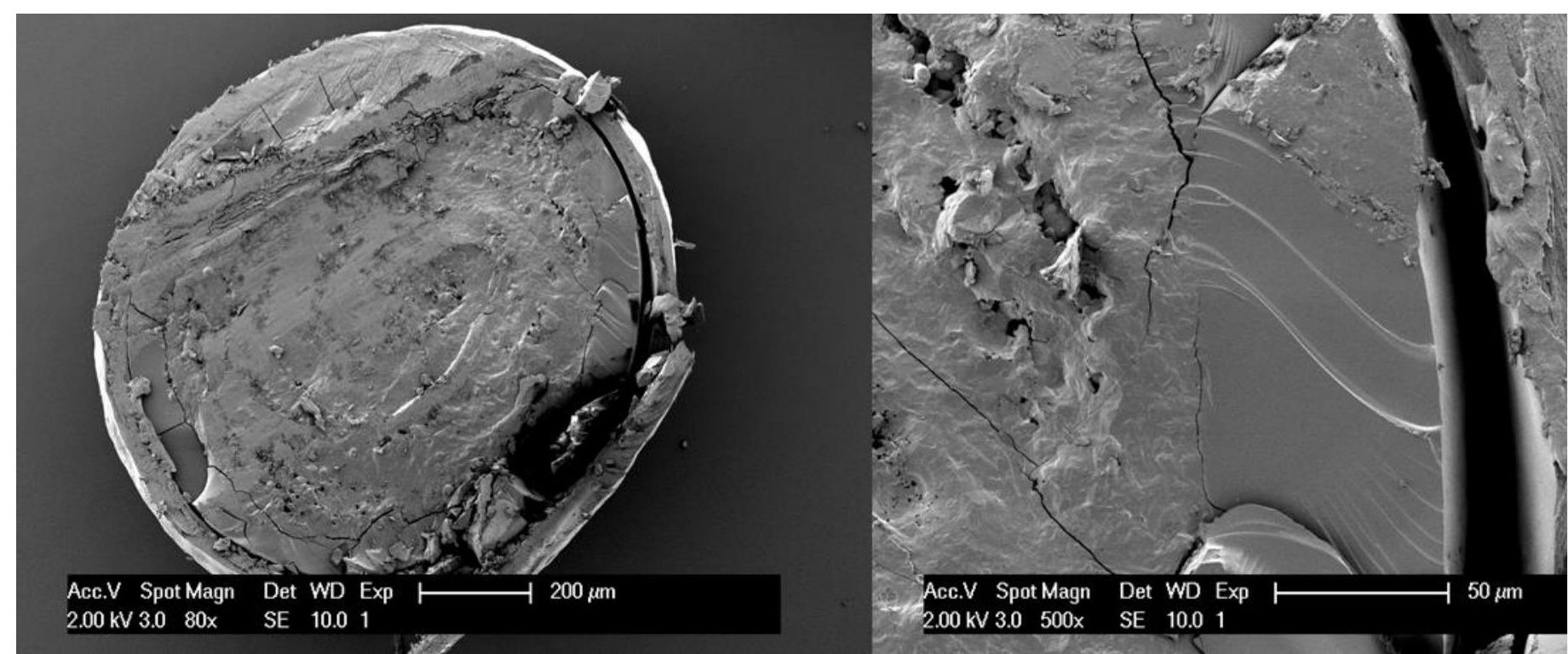

Figure B: SEM micrograph of a bead with INDO-PVP glass solution and EC-TEC top coating (left) and a detailed view on the coating layers (right).
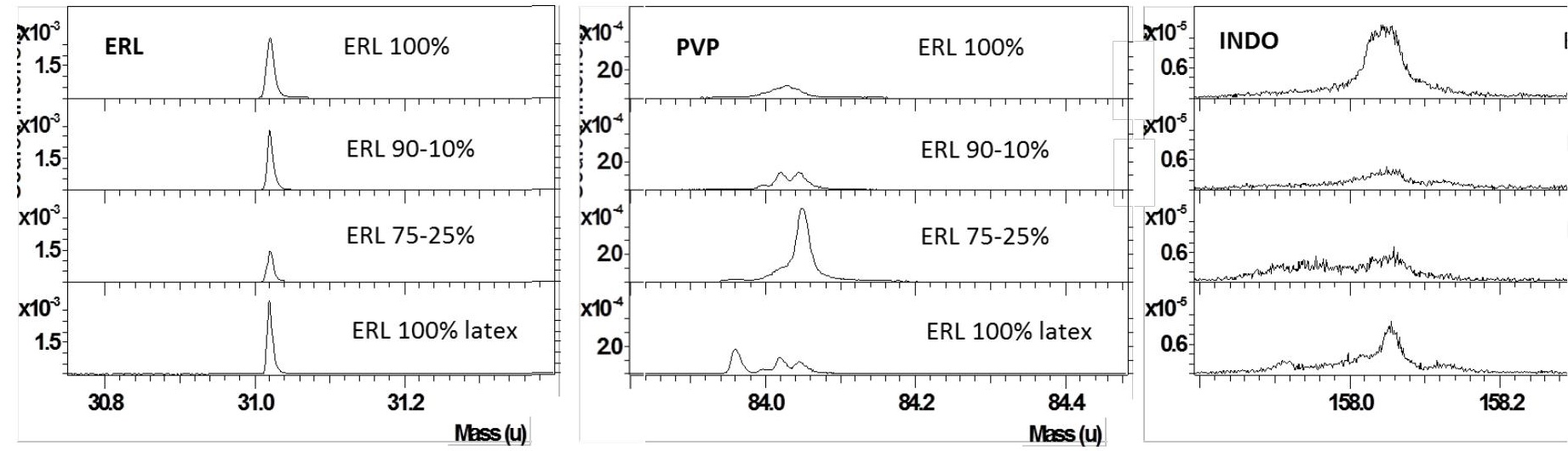

Figure C: ToF-SIMS ion spectra of ERL based outer layer coatings at the marker ion masses of ERL, PVP and INDO
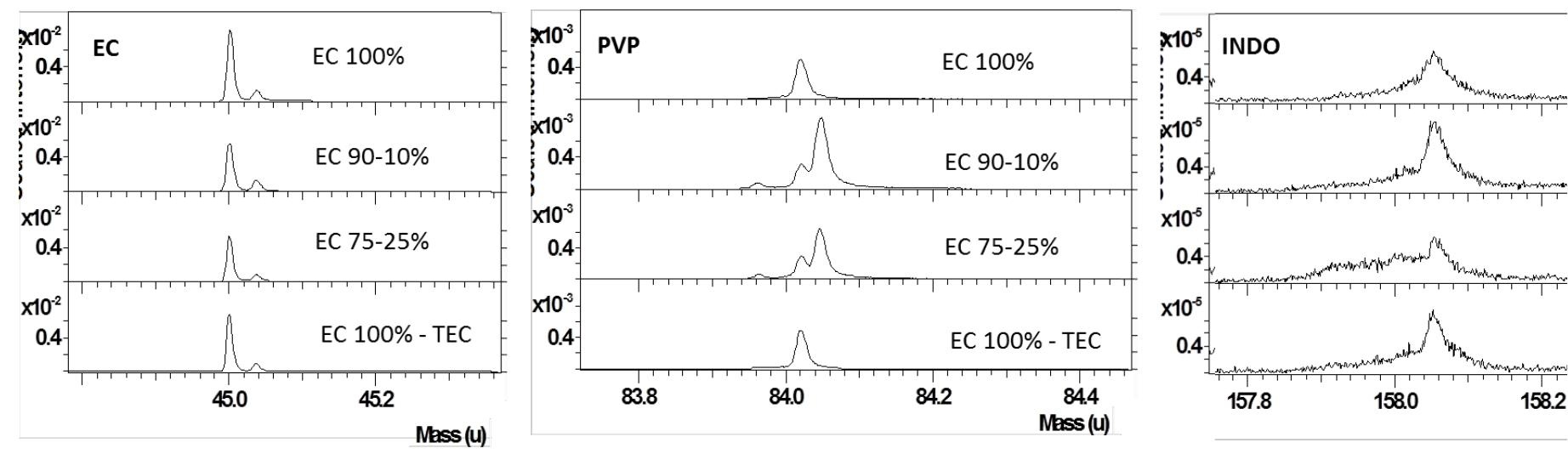

Figure D: ToF-SIMS ion spectra of EC based outer layer coatings at the marker ion masses of EC, PVP and INDO 

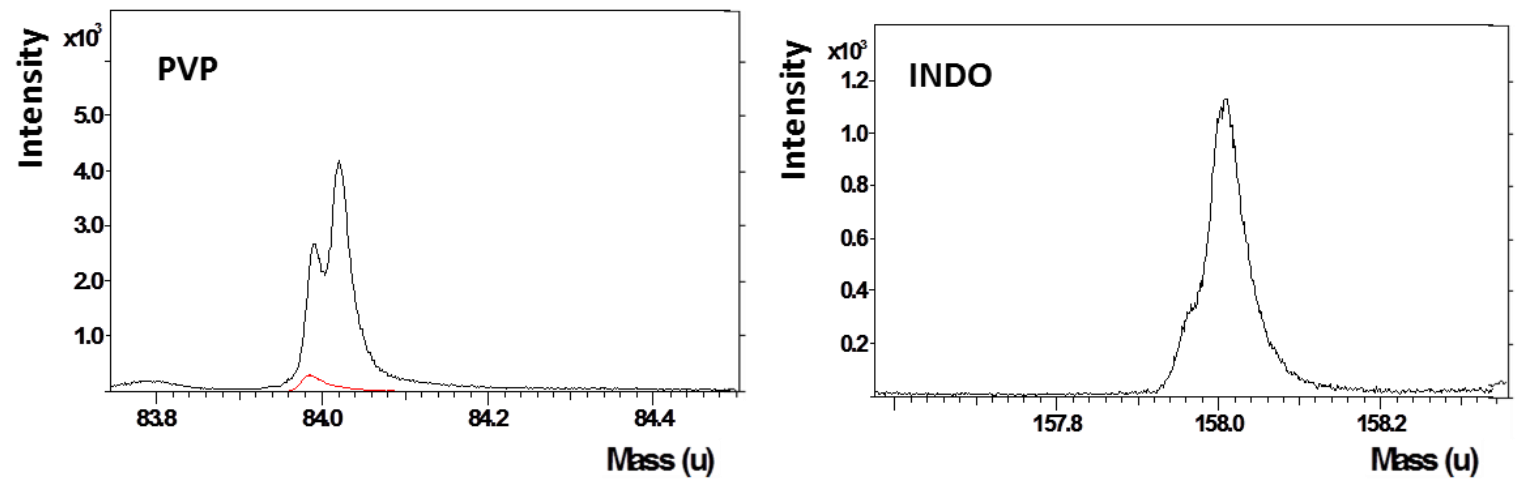

Figure E: ToF-SIMS ion spectra of INDO-PVP glass solution coating cross sections at the marker ion masses of PVP, INDO and sucrose.

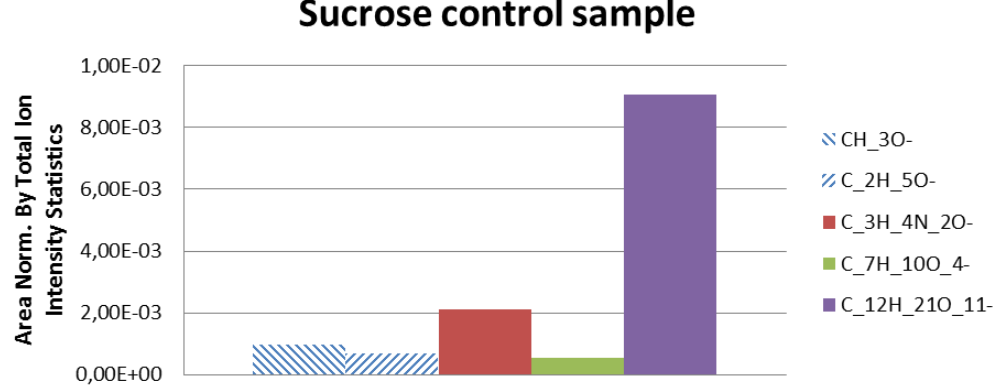

2

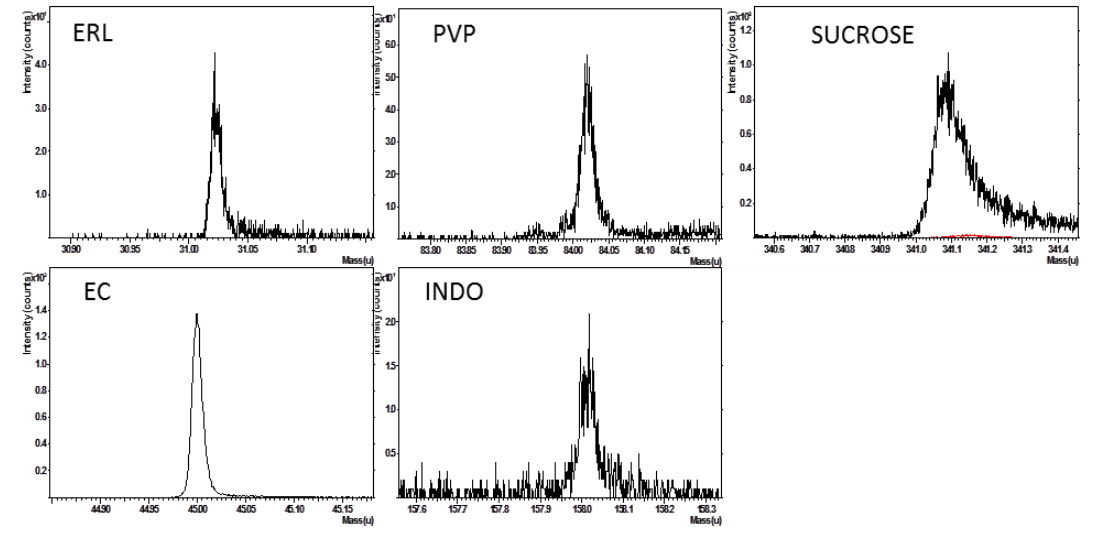

Figure F: Secondary ion spectra (1) and intensities (2) for the sucrose control sample. EC and ERL marker intensity is represented in striped blue bars, PVP marker intensity is a red bar, INDO marker intensity is a green bar and sucrose marker intensity is a purple bar. Ion intensities area is normalized by total ion statistics. 

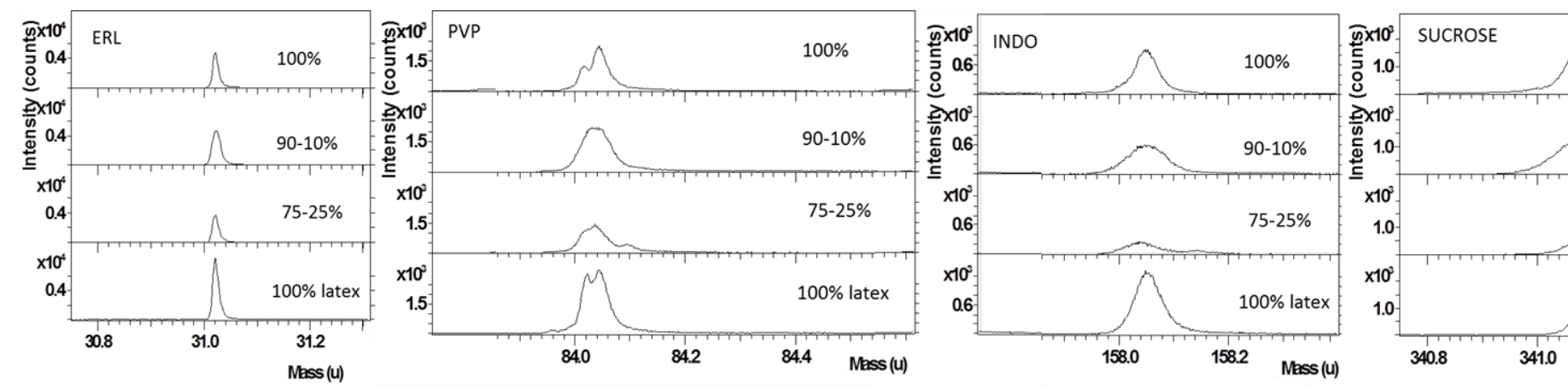

Figure G: ToF-SIMS ion spectra of ERL based outer layer coating cross-sections at the marker ion masses of ERL, PVP, INDO and Sucrose
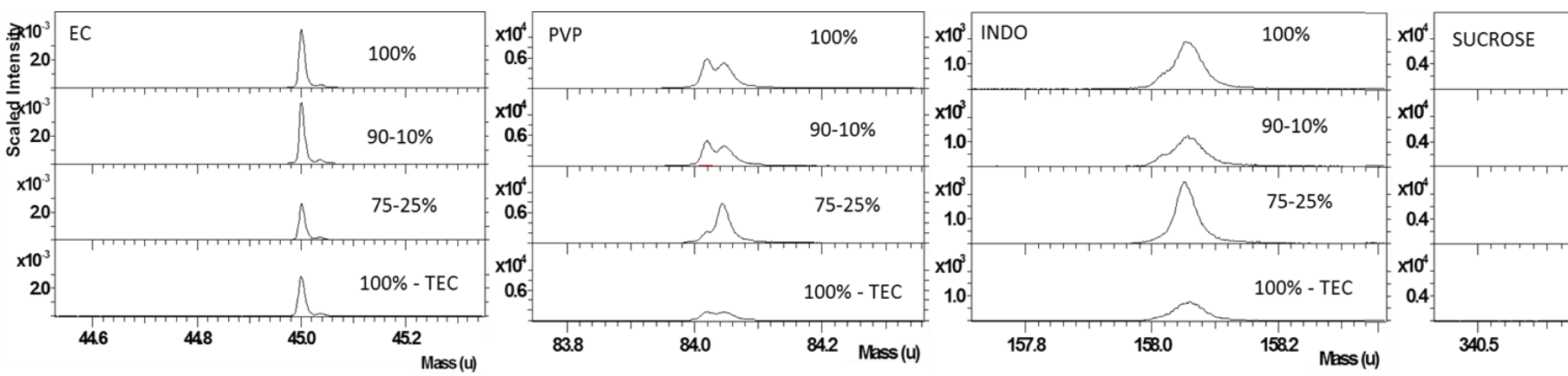

Figure H: ToF-SIMS ion spectra of EC based outer layer coating cross-sections at the marker ion masses of EC, PVP, INDO and Sucrose

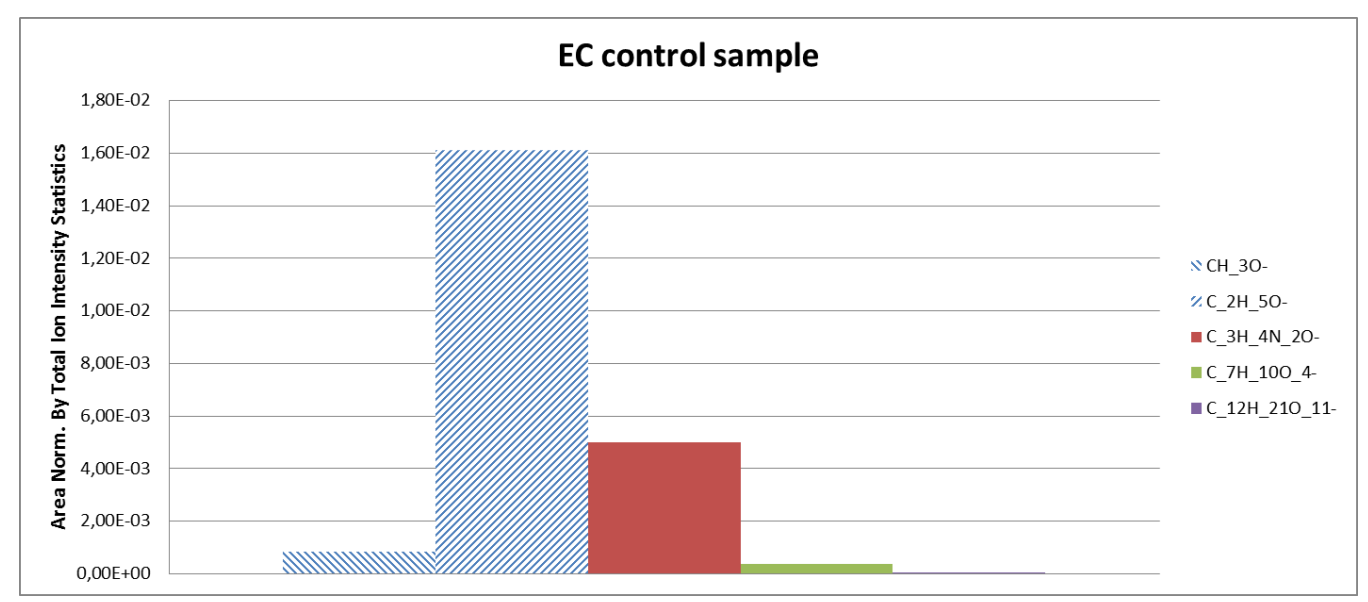

Figure I: Secondary ion intensities for the sucrose control sample. EC and ERL marker intensity is represented in striped blue bars, PVP marker intensity is a red bar, INDO marker intensity is a green bar and sucrose marker intensity is a purple bar. Ion intensities area is normalized by total ion statistics. 


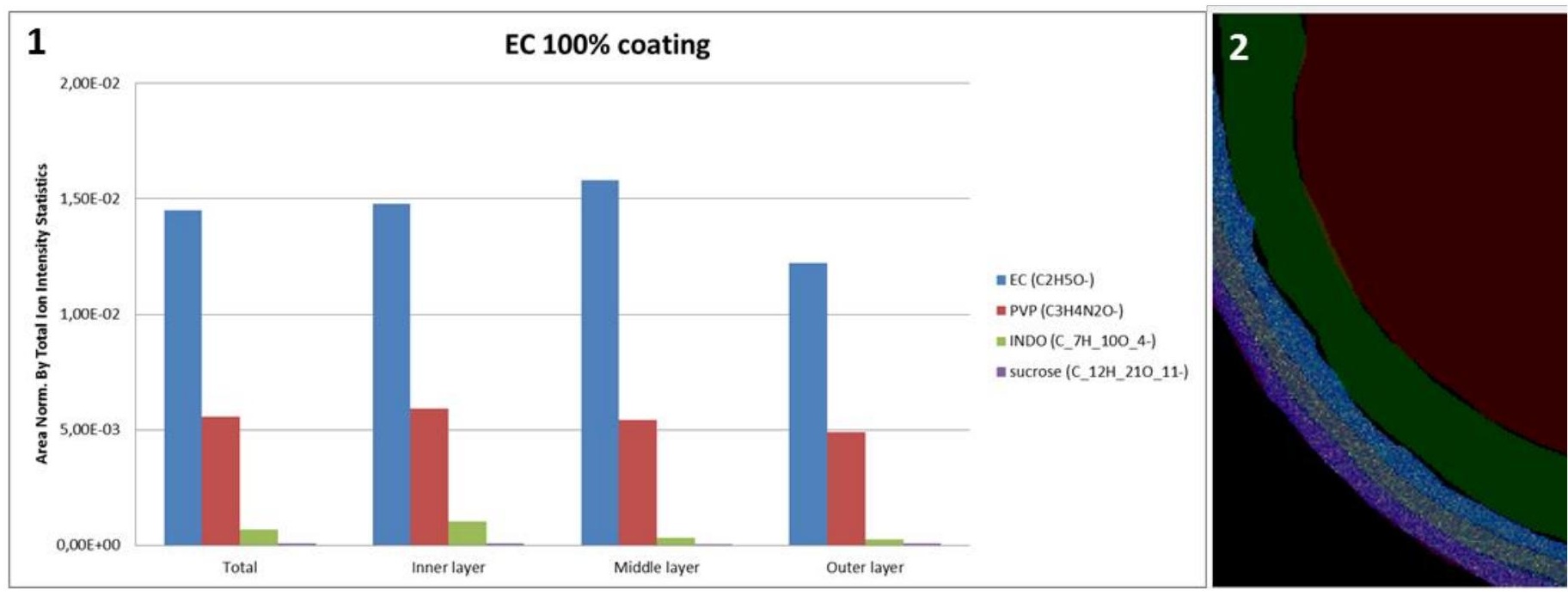

Figure I: Secondary ion intensities (1) of the . EC marker intensity is represented in blue bars, PVP marker intensity in red bars, INDO marker intensity in green bars and sucrose marker intensity in purple bars. Ion intensities area is normalized by total ion statistics. The region of interest creation (2), the purple colored region represents the outer layer of the EC 100\% coating, the yellow colored region the middle layer and the blue colored region the inner layer. 